CERN-TH/96-231

DESY 96-170

hep-ph/9611272

\title{
Soft Gluon Radiation in Higgs Boson Production at THE LHC
}

\author{
Michael KrämeR ${ }^{a}$, 凹, ERIC Laenen ${ }^{b}$ And Michael Spira ${ }^{b}$ \\ ${ }^{a}$ Deutsches Elektronen-Synchrotron DESY, D-22603 Hamburg, FRG \\ ${ }^{b}$ CERN TH-Division, CH-1211 Geneve 23, Switzerland
}

\begin{abstract}
We examine the contributions of soft gluons to the Higgs production cross section at the LHC in the Standard Model and its minimal supersymmetric extension. The soft gluon radiation effects of this reaction share many features with the Drell-Yan process, but arise at lowest order from a purely gluonic initial state. We provide an extension of the conventional soft gluon resummation formalism to include a new class of contributions which we argue to be universal, and resum these and the usual Sudakov effects to all orders. The effect of these new terms is striking: only if they are included, does the expansion of the resummed cross section to next-to-leading order reproduce the exact result to within a few percent for the full range of Higgs boson masses. We use our resummed cross section to derive next-to-next-to-leading order results, and their scale dependence. Moreover, we demonstrate the importance of including the novel contributions in the resummed Drell-Yan process.
\end{abstract}

CERN-TH/96-231

DESY 96-170

hep-ph/9611272

November 1996

\footnotetext{
${ }^{1}$ Present address: Rutherford Appleton Laboratory, Chilton, Didcot, OX11 0QX, England
} 


\section{Introduction}

The search for Higgs particles [1] is one of the most important endeavors for future high energy $e^{+} e^{-}$and hadron collider experiments. The Higgs boson is the only particle of the Standard Model (SM) which has not been discovered so far. The direct search at the LEP1 experiments via the process $e^{+} e^{-} \rightarrow Z^{*} H$ yields a lower bound on the Higgs mass of $65.2 \mathrm{GeV}$ [2]. Theoretical consistency restricts the Higgs mass to be smaller than $\sim 700 \mathrm{GeV}$ [3]. The dominant Higgs production mechanism at the LHC, a $p p$ collider with a c.m. energy of $14 \mathrm{TeV}$, is the gluon fusion process $g g \rightarrow H$ which is mediated by a heavy quark triangle loop at lowest order [4]. As an important step to increase the theoretical precision the two-loop QCD corrections have been calculated, resulting in a significant increase of the predicted total cross section by about $50-100 \%$ [5:6]. The dependence on the unphysical renormalization and factorization scales decreased considerably by including these next-to-leadingorder (NLO) corrections, resulting in an estimate of about $15 \%$ for the remaining scale sensitivity [5]. It is important to note, and we will demonstrate, that the NLO corrections are dominated by soft gluon radiation effects.

The minimal supersymmetric extension of the Standard Model (MSSM) is among the most attractive extensions of the SM. It requires the introduction of two Higgs doublets leading to the existence of five scalar Higgs particles, two scalar CP-even $h, H$, one pseudoscalar CP-odd $A$ and two charged bosons $H^{ \pm}$. This Higgs sector can be described by fixing two parameters, which are usually chosen to be $\operatorname{tg} \beta$, the ratio of the two vacuum expectation values, and the pseudoscalar Higgs mass $M_{A}$. Including higher order corrections to the Higgs masses and couplings up to the two-loop level, the mass of the lightest scalar Higgs particle $h$ is restricted to be smaller than $\sim 130$ $\mathrm{GeV}$ [7]. The direct search at LEP1 sets lower bounds of about $45 \mathrm{GeV}$ on the masses of the MSSM Higgs bosons [2]. The dominant neutral Higgs production mechanisms at the LHC are the gluon fusion processes $g g \rightarrow h, H, A$ and the associated production with a $b \bar{b}$ pair $g g, q \bar{q} \rightarrow b \bar{b} h, b \bar{b} H, b \bar{b} A$ which becomes important only for large $\operatorname{tg} \beta[8]$. The coupling of the neutral Higgs particles to gluons is again mediated by top and bottom loops, with the latter providing the dominant contribution for large $\operatorname{tg} \beta$, and squark loops, if their masses are smaller than about $400 \mathrm{GeV}$ [9]. (In this paper we shall assume the squark masses to be $1 \mathrm{TeV}$, so that squark loops can safely be neglected.) The two-loop (NLO) QCD corrections to the gluon fusion mechanism have also been calculated [5,9] and conclusions completely analogous to the SM case emerge. Soft gluon radiation effects again provide the dominant contribution to these corrections, for small $\operatorname{tg} \beta$. For large $\operatorname{tg} \beta$ bottom mass effects will be of similar size due to the dominance of the bottom quark loops and we will not consider this regime in this paper.

In previous analyses the resummation of soft gluon radiation in the transverse 
momentum distribution of the Higgs bosons has been performed [10], which are significant at small $p_{T}$. We consider universal soft gluon effects on the total production cross section and demonstrate that these dominate the NLO corrections both in the $\mathrm{SM}$ and the MSSM for small $\operatorname{tg} \beta$. The study of these effects in higher orders, and their resummation to all orders is our purpose in this paper.

Although our main focus is Higgs production, we will consider soft gluon effects in the Drell-Yan process for comparison. Higgs production shares many features with this reaction, apart from the species of leading initial state partons, e.g. it also proceeds at lowest order via a color singlet hard scattering process, and is a $2 \rightarrow 1$ process at lowest order. The Drell-Yan process has been studied by performing exact perturbative QCD calculations up to next-to-next-to-leading order (NNLO) in Ref. [11] and in the context of soft gluon resummation in [12]. In this paper we present an extension of the conventional soft gluon resummation formalism, in which we use the Drell-Yan reaction to gauge its quality and importance. We then apply the extension to Higgs production to derive the first estimates of NNLO effects. These estimates are important in view of the size of the NLO corrections.

The paper is organized as follows: In section 2 we describe the construction of the resummed cross section and the extension of the soft gluon resummation formalism. In section 3 we present NLO and NNLO results from the expansion of the resummed cross sections for Higgs production and the Drell-Yan process. We conclude and present an outlook in section 4 .

\section{The Resummed Exponent}

In this section we derive the resummed partonic cross section for Higgs boson production via gluon fusion. In order to set the stage, we must first discuss some preliminary approximations to the exact NLO calculation in Ref. [5].

In the Standard Model, the leading order (LO) process consists of gluon fusion into a Higgs boson via a heavy quark triangle loop, see Fig. 1. Because the Higgs coupling to fermions is proportional to the fermion mass, the top quark strongly dominates this coupling, constituting about $90 \%$ of the total coupling. Our first approximation is to neglect the quark-antiquark and quark-gluon channels as these contribute at next-to-leading order (NLO) to the full cross section with less than $10 \%$ [5].

The exact NLO calculation in [5] was performed for the general massive case, i.e. all masses were taken into account explicitly. However, the following very useful approximation was identified, involving the heavy top mass limit of the calculation. 


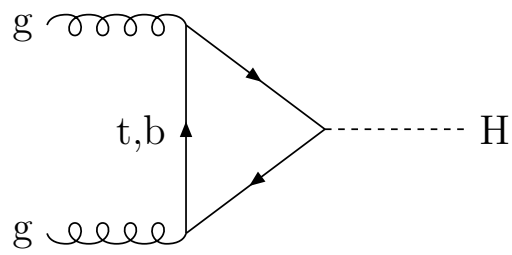

Figure 1: Higgs boson production via gluon fusion mediated by top-and bottom quark loops

Let us define the (NLO) K-factorf by

$$
K_{N L O}^{t+b}\left(\tau_{t}, \tau_{b}\right) \equiv \frac{\sigma_{N L O}\left(\tau_{t}, \tau_{b}\right)}{\sigma_{L O}\left(\tau_{t}, \tau_{b}\right)}
$$

where $\sigma_{L O / N L O}\left(\tau_{t}, \tau_{b}\right)$ denotes the hadronic gluon-fusion cross section for the general massive case, calculated exactly in $\mathrm{LO} / \mathrm{NLO}$, and the scaling variables are defined by $\tau_{Q}=4 m_{Q}^{2} / M_{H}^{2}(Q=t, b)$.

In Figs. 2 and 3 we compare $\sigma_{N L O}\left(\tau_{t}, \tau_{b}\right)$ with the approximation

$$
K_{N L O}^{t}(\infty) \times \sigma_{L O}\left(\tau_{t}, \tau_{b}\right)
$$

for scalar and pseudoscalar Higgs boson production, where the K-factor $K_{N L O}^{t}(\infty)$ takes into account the top quark contribution to the relative QCD corrections only, in the limit of a heavy top quark. We observe that the approximation (2) is accurate to within $10 \%$ for the full Higgs mass range $M_{H} \gtrsim 65 \mathrm{GeV}$ of the SM Higgs boson as well as the pseudoscalar Higgs particle of the MSSM for small $\operatorname{tg} \beta$ [At the $t \bar{t}$ threshold $M_{A}=2 m_{t}$ the pseudoscalar cross section develops a Coulomb singularity so that perturbation theory is not valid in a small margin around this value for the pseudoscalar mass $M_{A}$ [5].]. The same accuracy of the approximation emerges for the two scalar Higgs particles of the MSSM for small $\operatorname{tg} \beta$. Our second approximation then consists of assuming that $K^{t}(\infty) \times \sigma_{L O}\left(\tau_{t}, \tau_{b}\right)$ is a valid approximation to $\sigma\left(\tau_{t}, \tau_{b}\right)$ for all orders, i.e. we will assume that the higher-order K-factor, when computed in the infinite top mass limit and combined with the massive LO cross section, will give a good approximation to the higher order cross section in the general massive case. In fact, we will see that at NLO the bulk of the K-factor is due to soft and collinear gluons, which do not resolve the effective coupling. The assumption that this persists

\footnotetext{
${ }^{2}$ It should be noted that we use here NLO parton densities and strong coupling $\alpha_{s}$ in the NLO cross sections and LO quantities in the LO cross sections for this "hadronic" K-factor. This leads to K-factors smaller than two in contrast with the "partonic" K-factor, which is defined with NLO parton densities and strong coupling also in the LO cross section.
} 


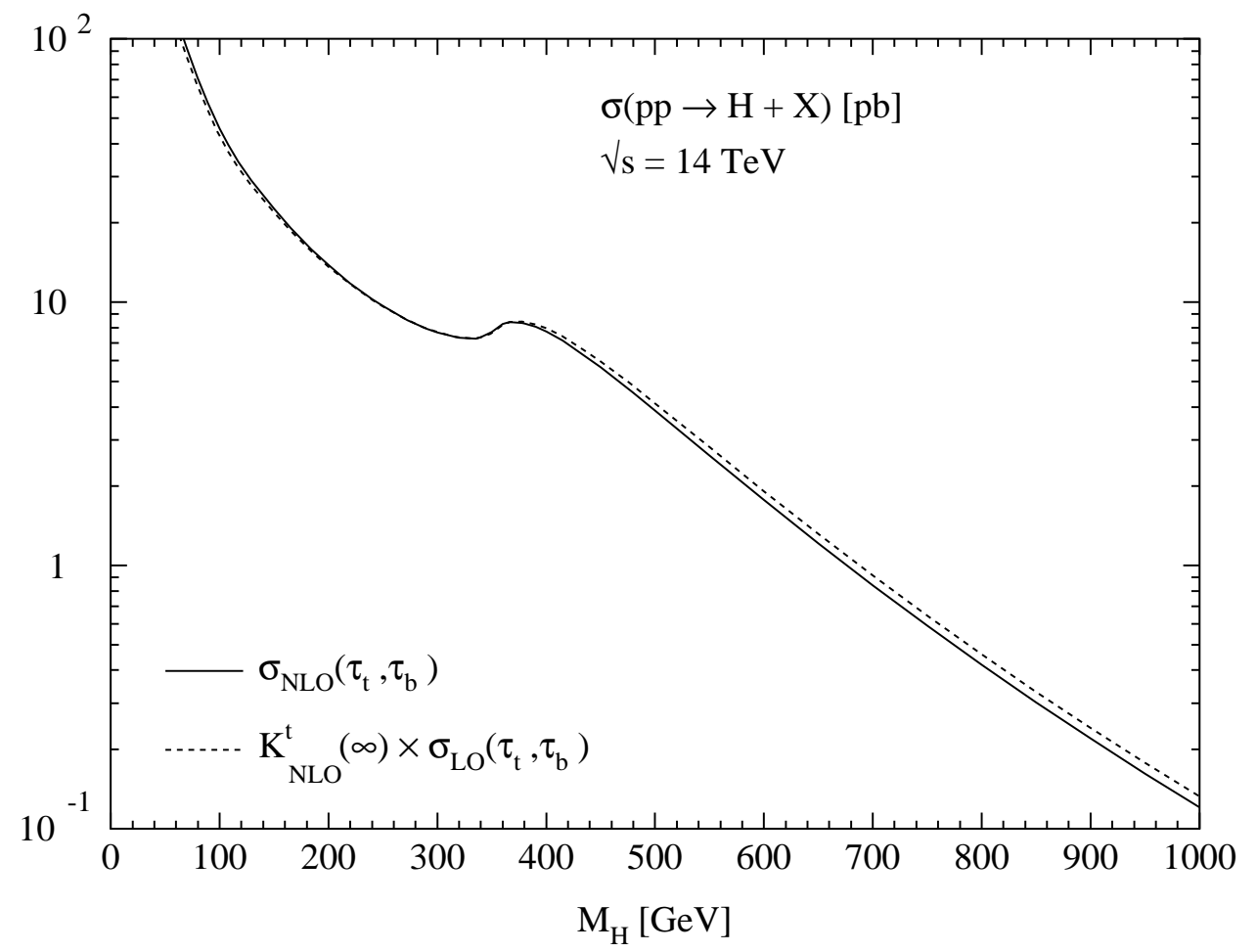

Figure 2: Exact and approximate results in the heavy top quark limit for the total SM Higgs production cross sections as a function of the Higgs mass $M_{H}$. The top mass has been chosen as $m_{t}=175 \mathrm{GeV}$ and the bottom mass as $m_{b}=5 \mathrm{GeV}$. CTEQ4M parton densities [13] with $N L O$ strong coupling $\left[\alpha_{s}\left(M_{Z}^{2}\right)=0.116\right]$ have been used.

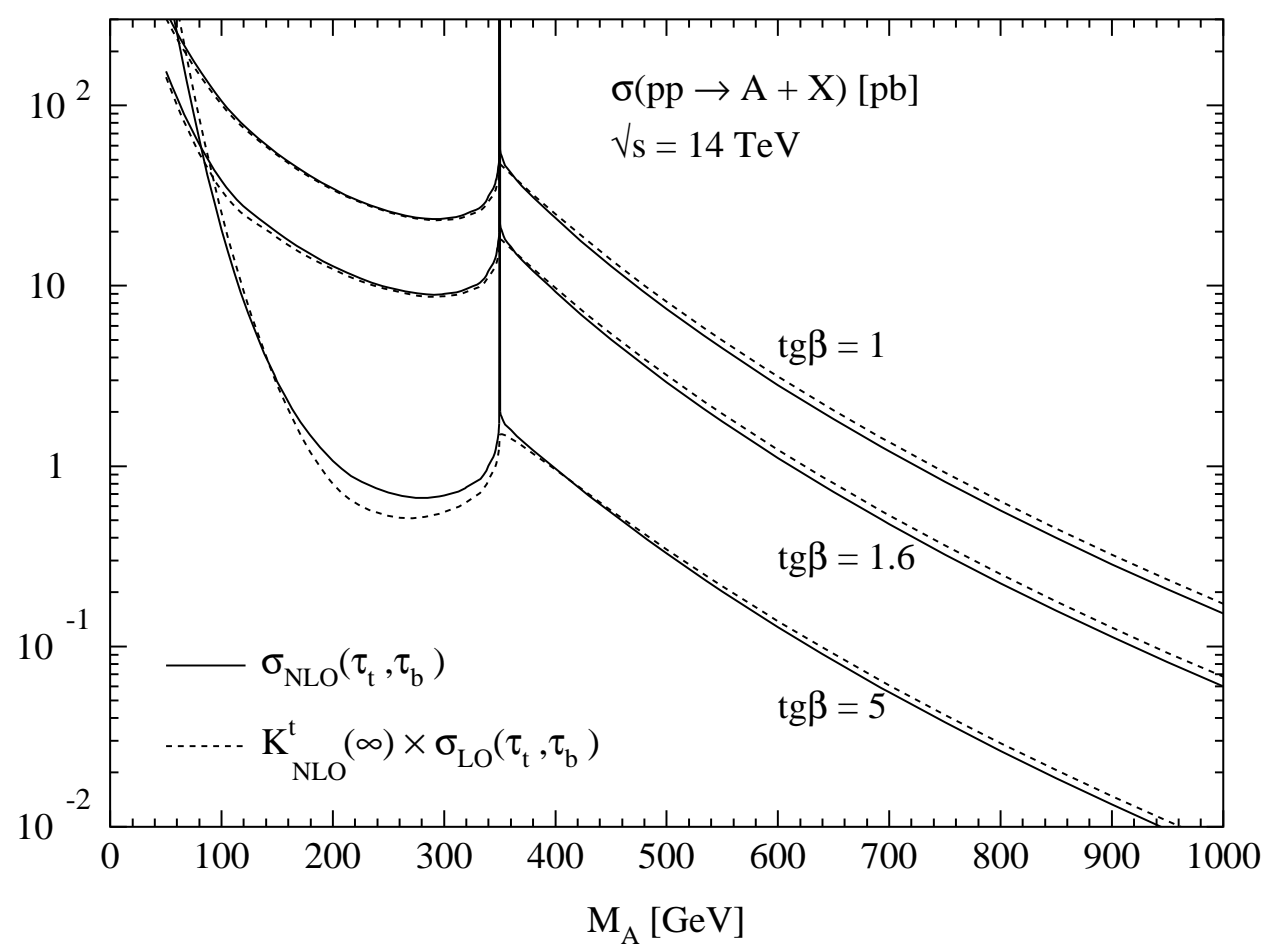

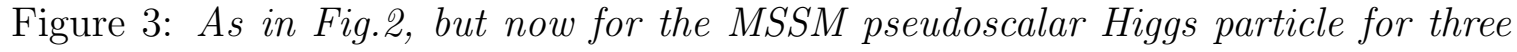
values of $\operatorname{tg} \beta$. 
to higher orders is supported by the validity of the infinite top mass approximation at NLO.

In the MSSM, the validity of these approximations depends strongly on the parameter $\operatorname{tg} \beta$. For $\operatorname{tg} \beta \lesssim 1.6$ the top quark contribution to the cross sections amounts to more than $70 \%$. The heavy top quark limit is thus a reasonable approximation in this regime. For large values of $\operatorname{tg} \beta$ the bottom loop contribution becomes significant so that the approximations are no longer valid. Still, the infinite top mass approximation deviates from the full NLO result, including bottom contributions, by less than $25 \%$ for $\operatorname{tg} \beta \lesssim 5$ as can be inferred from Fig. 3 .

We are now ready for the construction of the resummed partonic cross section, for which we will employ the methods of Ref. [14]. In order to retain similarity to the Drell-Yan case, we will denote the Higgs mass squared with $Q^{2}$ throughout the text of this paper.

In the approximations outlined above, the regularized total Higgs production cross sections may be written in $d=4-2 \epsilon$ dimensions as $[\phi=h, H, A]$

$$
\sigma^{\phi}\left(\tau_{\phi}, Q^{2}, \mu^{2}\right)=\int_{\tau_{\phi}}^{1} d x_{1} \int_{\tau_{\phi} / x_{1}}^{1} d x_{2} g\left(x_{1}\right) g\left(x_{2}\right) \hat{\sigma}_{g g}^{\phi}\left(z, Q^{2}, \mu^{2}, \epsilon\right)
$$

or, in terms of moments,

$$
\sigma^{\phi}\left(N, Q^{2}, \mu^{2}\right)=\int_{0}^{1} d \tau_{\phi} \tau_{\phi}^{N-1} \sigma^{\phi}\left(\tau_{\phi}, Q^{2}, \mu^{2}\right)=g^{2}(N+1) \hat{\sigma}_{g g}^{\phi}\left(N, Q^{2}, \mu^{2}, \epsilon\right)
$$

where $\tau_{\phi}=Q^{2} / S, z=\tau_{\phi} /\left(x_{1} x_{2}\right), S$ is the hadronic c.m. energy squared, $\mu$ is the dimensional regularization scale, and $g(x)$ is the bare gluon distribution function. Note that the definition is such that the dependence on the moment variable in the parton densities is shifted by one unit compared to the dependence of the partonic cross section. In this way we remove an overall $1 / z$ factor, emphasizing the soft gluon contribution to the partonic cross section.

In the approximations discussed in the beginning of this section, the $d$-dimensional partonic cross sections can be cast into the form

$$
\hat{\sigma}_{g g}^{\phi}=\sigma_{0}^{\phi} \kappa_{\phi} \rho_{\phi}\left(z, Q^{2} / \mu^{2}, \epsilon\right)
$$

with the coefficients

$$
\begin{aligned}
\sigma_{0}^{h, H} & =g_{t}^{h, H} \frac{G_{F} \alpha_{s, B}^{2} N_{C} C_{F}}{1152 \sqrt{2} \pi} \frac{\Gamma^{2}(1+\epsilon)}{1-\epsilon}\left(\frac{4 \pi}{m_{t}^{2}}\right)^{2 \epsilon}, \\
\sigma_{0}^{A} & =g_{t}^{A} \frac{G_{F} \alpha_{s, B}^{2} N_{C} C_{F}}{512 \sqrt{2} \pi} \frac{\Gamma^{2}(1+\epsilon)}{1-\epsilon}\left(\frac{4 \pi}{m_{t}^{2}}\right)^{2 \epsilon},
\end{aligned}
$$

where $\alpha_{s, B}$ is the bare strong coupling constant (with dimension $2 \epsilon$ ) and $g_{t}^{\phi}(\phi=$ $h, H, A)$ denote the modified top Yukawa couplings normalized to the SM coupling, 
which are given in [5]. The factor $\kappa_{\phi}$ in eq. (5) stems from the effective coupling of the Higgs boson to gluons in the heavy top quark limit, which can be obtained by means of low energy theorems [5,15]. They are given $3^{3}$ by [5, 16]

$$
\begin{aligned}
\kappa_{h, H} & =\left\{\frac{3 \pi}{\left[\alpha_{s}^{(5)}\left(\bar{m}_{t}^{2}\right)\right]^{2}} \frac{\beta_{t}\left[\alpha_{s}^{(6)}\left(\bar{m}_{t}^{2}\right)\right]}{1+\gamma_{m}\left[\alpha_{s}^{(6)}\left(\bar{m}_{t}^{2}\right)\right]}\left(\frac{\alpha_{s}^{(5)}\left(\bar{m}_{t}^{2}\right)}{\alpha_{s}^{(5)}\left(M_{h, H}^{2}\right)}\right)^{2} \frac{\beta\left[\alpha_{s}^{(5)}\left(M_{h, H}^{2}\right)\right]}{\beta\left[\alpha_{s}^{(5)}\left(\bar{m}_{t}^{2}\right)\right]}\right\}^{2} \\
\kappa_{A} & =1
\end{aligned}
$$

where $\alpha_{s}^{\left(n_{f}\right)}\left(n_{f}=5,6\right)$ is the strong coupling constant in the $\overline{\mathrm{MS}}$ scheme including $n_{f}$ flavors in the evolution; the couplings for 5 and 6 flavors are related by 18

$$
\alpha_{s}^{(5)}\left(\bar{m}_{t}^{2}\right)=\alpha_{s}^{(6)}\left(\bar{m}_{t}^{2}\right)\left\{1+\frac{11}{72}\left(\frac{\alpha_{s}^{(6)}\left(\bar{m}_{t}^{2}\right)}{\pi}\right)^{2}\right\}
$$

$\beta\left(\alpha_{s}\right)$ denotes the QCD $\beta$ function and $\beta_{t}\left(\alpha_{s}\right)$ its top quark contribution $甲$, which is given by $18,20\left[n_{f}=5\right.$ is the number of light flavors]

$$
\frac{3 \pi}{\alpha_{s}^{2}} \beta_{t}\left(\alpha_{s}\right)=1+\frac{19}{4} \frac{\alpha_{s}}{\pi}+\frac{6793-281 n_{f}}{288} \frac{\alpha_{s}^{2}}{\pi^{2}}+\mathcal{O}\left(\alpha_{s}^{3}\right)
$$

$\gamma_{m}\left(\alpha_{s}\right)$ is the anomalous mass dimension including 6 flavors, which can be expressed as [21]

$$
\gamma_{m}\left(\alpha_{s}\right)=2 \frac{\alpha_{s}}{\pi}+\left(\frac{101}{12}-\frac{5}{18}\left[n_{f}+1\right]\right) \frac{\alpha_{s}^{2}}{\pi^{2}}+\mathcal{O}\left(\alpha_{s}^{3}\right)
$$

Using these expansions the effective scalar couplings $\kappa_{h, H}$ are given by

$$
\begin{aligned}
\kappa_{h, H}= & 1+\frac{11}{2} \frac{\alpha_{s}^{(5)}\left(m_{t}^{2}\right)}{\pi}+\frac{3866-201 n_{f}}{144}\left(\frac{\alpha_{s}^{(5)}\left(m_{t}^{2}\right)}{\pi}\right)^{2} \\
& +\frac{153-19 n_{f}}{33-2 n_{f}} \frac{\alpha_{s}^{(5)}\left(M_{h, H}^{2}\right)-\alpha_{s}^{(5)}\left(m_{t}^{2}\right)}{\pi}+\mathcal{O}\left(\alpha_{s}^{3}\right)
\end{aligned}
$$

where $m_{t}$ denotes the pole mass of the top quark. The scale dependence of the strong coupling in the lowest order cross section eq. (7) and the factor $\rho_{\phi}$ eq. (5) includes 5 light flavors, i.e. the top quark is decoupled. In the rest of the paper we identify

$$
\alpha_{s}\left(\mu^{2}\right) \equiv \alpha_{s}^{(5)}\left(\mu^{2}\right)
$$

\footnotetext{
${ }^{3}$ The last two factors in the large bracket originate from the anomalous dimension of the gluon operators [16]. The top mass $\bar{m}_{t}$ denotes the scale invariant $\overline{\mathrm{MS}}$ mass $\bar{m}_{t}=\bar{m}_{t}\left(\bar{m}_{t}\right)$. We would like to thank the authors of Ref. [17] for pointing out two errors in our treatment of the strong coupling in eq.(8) and the anomalous mass dimension of eq.(12) in an earlier version of this paper. The numerical size of these errors is about $0.03 \%$ and thus negligible.

${ }^{4}$ The factors $\kappa_{h, H}$ include the top quark contribution at vanishing momentum transfer, which differs from the top quark contribution to the $\overline{\mathrm{MS}} \beta$ function by a finite amount at $\mathcal{O}\left(\alpha_{s}^{4}\right)$ [19].

${ }^{5}$ This result agrees with Ref. [17].
} 
Note further that the expression (5) is not yet finite for $d \rightarrow 4$; mass factorization and renormalization of the bare coupling in the Born cross section will be carried out after resummation. In eq. (5) we denote the correction factors by $\rho_{\phi}\left(z, Q^{2} / \mu^{2}, \epsilon\right)$, which are defined in the infinite mass limit without the factorizing corrections $\kappa_{\phi}$ to the effective coupling. They may be expanded as

$$
\rho_{\phi}\left(z, Q^{2} / \mu^{2}, \alpha\left(\mu^{2}\right), \epsilon\right)=\sum_{n=0}^{\infty} \alpha^{n}\left(\mu^{2}\right) \rho_{\phi}^{(n)}\left(z, Q^{2} / \mu^{2}, \epsilon\right)
$$

where we define, for the sake of convenience,

$$
\alpha\left(\mu^{2}\right) \equiv \frac{\alpha_{s}\left(\mu^{2}\right)}{\pi} .
$$

Here $\alpha_{s}\left(\mu^{2}\right)$ is the renormalized strong coupling and we have chosen the renormalization scale equal to $\mu$ for the moment. From Ref. [5,6] we can derive the following expression for the first two coefficients of the SM Higgs correction factor

$$
\begin{aligned}
\rho_{\phi}^{(0)}\left(z, Q^{2} / \mu^{2}, \epsilon\right) & =\delta(1-z) \\
\rho_{h, H}^{(1)}\left(z, Q^{2} / \mu^{2}, \epsilon\right) & =\left(\frac{\mu^{2}}{Q^{2}}\right)^{\epsilon} C_{A}\left\{-\frac{z^{\epsilon}}{\epsilon}\left[\frac{1+z^{4}+(1-z)^{4}}{(1-z)^{1+2 \epsilon}}\right]_{+}\right. \\
& \left.+\delta(1-z)\left(\frac{11}{6 \epsilon}+\frac{203}{36}+\frac{\pi^{2}}{3}\right)-\frac{11}{6} z^{\epsilon}(1-z)^{3-2 \epsilon}\right\} \\
\rho_{A}^{(1)} & =\rho_{h, H}^{(1)}+2\left(\frac{\mu^{2}}{Q^{2}}\right)^{\epsilon} C_{A} \delta(1-z)
\end{aligned}
$$

Note that we have implicitly redefined the scale $\mu$ by $\mu^{2} \rightarrow \mu^{2} \exp \left[-\left(\ln (4 \pi)-\gamma_{E}\right)\right]$ to eliminate factors $(4 \pi)^{\epsilon}$ and $\Gamma(1-\epsilon) / \Gamma(1-2 \epsilon)$. The plus distribution in eq. (18) is as usual defined by

$$
\int_{x}^{1} d z g(z)[f(z)]_{+}=\int_{x}^{1} d z(g(z)-g(1)) f(z)-g(1) \int_{0}^{x} d z f(z) .
$$

We will now construct a resummed expression for $\rho_{\phi}\left(z, Q^{2} / \mu^{2}, \alpha, \epsilon\right)$ by means of the methods described in Ref. [14]. Near the elastic edge of phase space the Higgs cross section in the infinite mass limit may be factorized into hard, soft and jet functions, in completely analogy with the Drell-Yan cross section. Following the arguments of Ref. [14] this leads to the Sudakov evolution equation

$$
Q^{2} \frac{d}{d Q^{2}} \rho_{\phi}\left(z, Q^{2} / \mu^{2}, \alpha\left(\mu^{2}\right), \epsilon\right)=\int_{z}^{1} \frac{d z^{\prime}}{z^{\prime}} W_{\phi}\left(z^{\prime}, Q^{2} / \mu^{2}, \alpha\left(\mu^{2}\right), \epsilon\right) \rho_{\phi}\left(z / z^{\prime}, Q^{2} / \mu^{2}, \alpha\left(\mu^{2}\right), \epsilon\right) .
$$

In order to solve eq. (21) we must impose a boundary condition. We will shortly argue [14] that we may use the boundary condition $\tilde{\rho}_{\phi}\left(N, Q^{2} / \mu^{2}=0, \alpha\left(\mu^{2}\right), \epsilon\right)=1$ for the moments, or in $z$-space

$$
\rho_{\phi}\left(z, Q^{2} / \mu^{2}=0, \alpha\left(\mu^{2}\right), \epsilon\right)=\delta(1-z) .
$$


The solution to eq. (21) is then, in Mellin space,

$$
\tilde{\rho}_{\phi}\left(N, Q^{2} / \mu^{2}, \alpha\left(\mu^{2}\right), \epsilon\right)=\exp \left[\int_{0}^{Q^{2}} \frac{d \xi^{2}}{\xi^{2}} \tilde{W}_{\phi}\left(N, \frac{\xi^{2}}{\mu^{2}}, \alpha\left(\mu^{2}\right), \epsilon\right)\right],
$$

where as above $d=4-2 \epsilon>4$. The formal Mellin inversion reads

$$
W_{\phi}\left(z, Q^{2} / \mu^{2}, \alpha\left(\mu^{2}\right), \epsilon\right)=\int_{-i \infty}^{i \infty} \frac{d N}{2 \pi i} N^{-z} \tilde{W}_{\phi}\left(N, Q^{2} / \mu^{2}, \alpha\left(\mu^{2}\right), \epsilon\right) .
$$

The solution (23) may be expressed as

$$
\begin{aligned}
\tilde{\rho}_{\phi}\left(N, Q^{2} / \mu^{2}, \alpha\left(\mu^{2}\right), \epsilon\right) & =\exp \left[\int _ { 0 } ^ { 1 } d z z ^ { N - 1 } \int _ { 0 } ^ { \nu Q ^ { 2 } / \mu ^ { 2 } } \frac { d \lambda } { \lambda } \left\{\alpha\left(\lambda, \alpha\left(\mu^{2}\right), \epsilon\right) W_{\phi}^{(1)}(z, 1 / \nu, \epsilon)\right.\right. \\
& \left.\left.+\alpha^{2}\left(\lambda, \alpha\left(\mu^{2}\right), \epsilon\right) W_{\phi}^{(2)}(z, 1 / \nu, \epsilon)+\mathcal{O}\left(\alpha^{3}\right)\right\}\right]
\end{aligned}
$$

with $\nu(z)$ an arbitrary function. Note that $\nu(z)$ is to be treated as part of any plus distributions in $W_{\phi}$, because it arises from the originally $z$-independent scale ratio. In deriving eq. (25) we have used the renormalization group invariance of the evolution kernel $W_{\phi}$. Note further that we have expanded the full evolution kernel $W_{\phi}\left(z, \xi^{2} / \mu^{2}, \alpha\left(\mu^{2}\right), \epsilon\right)$ in the $d$-dimensional running coupling constant, with $\lambda=\nu(z) \xi^{2} / \mu^{2}$. The defining equation of the $d$-dimensional running coupling is

$$
\lambda^{1-\epsilon} \frac{\partial\left[\lambda^{\epsilon} \alpha\left(\lambda, \alpha\left(\mu^{2}\right), \epsilon\right)\right]}{\partial \lambda}=-b_{2} \alpha^{2}\left(\lambda, \alpha\left(\mu^{2}\right), \epsilon\right)-b_{3} \alpha^{3}\left(\lambda, \alpha\left(\mu^{2}\right), \epsilon\right),
$$

with the boundary condition $\alpha\left(1, \alpha\left(\mu^{2}\right), \epsilon\right)=\alpha\left(\mu^{2}\right)$. Here $b_{2}=\left(11 C_{A}-2 n_{f}\right) / 12$ and $b_{3}=34 C_{A}^{2} / 48-\left(20 C_{A} / 3+4 C_{F}\right) n_{f} / 32$. The solution, linearized in $b_{3}$, is

$$
\lambda^{\epsilon} \alpha\left(\lambda, \alpha\left(\mu^{2}\right), \epsilon\right)=\frac{\alpha\left(\mu^{2}\right)}{1-\gamma\left(\lambda^{\epsilon}, \epsilon\right) \alpha\left(\mu^{2}\right)}+\frac{b_{3}}{b_{2}} \frac{\alpha^{2}\left(\mu^{2}\right)}{\left[1-\gamma\left(\lambda^{\epsilon}, \epsilon\right) \alpha\left(\mu^{2}\right)\right]^{2}} f\left(\lambda^{\epsilon}, \alpha\left(\mu^{2}\right), \epsilon\right)
$$

with $\gamma\left(\lambda^{\epsilon}, \epsilon\right) \equiv \frac{b_{2}}{\epsilon}\left(\lambda^{-\epsilon}-1\right), f\left(\lambda^{\epsilon}, \alpha, \epsilon\right)=1-\lambda^{-\epsilon}-\left(1+\frac{\epsilon}{b_{2} \alpha}\right) \ln \left[1-\gamma\left(\lambda^{\epsilon}, \epsilon\right) \alpha\right]$. We shall now justify the boundary condition in eq. (22). To this end, note that for $\epsilon<0(d>4)$, the dimensionally-continued running coupling vanishes at zero scale, $\alpha\left(0, \alpha\left(\mu_{1}^{2}\right), \epsilon\right)=0$, order by order in its perturbative expansion in the coupling $\alpha\left(\mu_{1}^{2}\right)$ evaluated at any nonzero scale $\mu_{1}$. Dimensionally-continued radiative corrections to $\rho_{\phi}$ therefore vanish at $Q^{2}=0$.

The functions $W_{\phi}^{(1)}, W_{\phi}^{(2)}$ can be determined by choosing $\nu=1$ in eq. (25) and expanding the Sudakov equation to second order in the $d$-dimensional running coupling

$$
\begin{gathered}
Q^{2} \frac{\partial}{\partial Q^{2}} \ln \tilde{\rho}_{\phi}\left(N, Q^{2} / \mu^{2}, \alpha\left(\mu^{2}\right), \epsilon\right)=\alpha\left(Q^{2} / \mu^{2}, \alpha\left(\mu^{2}\right), \epsilon\right) \tilde{W}_{\phi}^{(1)}(N, 1, \epsilon) \\
+\alpha^{2}\left(Q^{2} / \mu^{2}, \alpha\left(\mu^{2}\right), \epsilon\right) \tilde{W}_{\phi}^{(2)}(N, 1, \epsilon)
\end{gathered}
$$


in terms of $\alpha_{s}\left(\mu^{2}\right)$, using (see eq. (27))

$$
\lambda^{\epsilon} \alpha\left(\lambda, \alpha\left(\mu^{2}\right), \epsilon\right)=\alpha\left(\mu^{2}\right)+\gamma\left(\lambda^{\epsilon}, \epsilon\right) \alpha^{2}\left(\mu^{2}\right)+\mathcal{O}\left(\alpha^{3}\right) .
$$

The resulting one- and two-loop coefficients of the evolution kernel can then be derived from low order calculations of the correction factors via

$$
\tilde{W}_{\phi}^{(1)}(N, 1, \epsilon)=\left(Q^{2} / \mu^{2}\right)^{\epsilon} Q^{2} \frac{\partial}{\partial Q^{2}} \tilde{\rho}_{\phi}^{(1)}\left(N, Q^{2} / \mu^{2}, \epsilon\right)
$$

and

$$
\begin{array}{r}
\tilde{W}_{\phi}^{(2)}(N, 1, \epsilon)=\left(Q^{2} / \mu^{2}\right)^{2 \epsilon}\left\{\frac{\partial}{\partial \ln Q^{2}}\left(\tilde{\rho}_{\phi}^{(2)}\left(N, Q^{2} / \mu^{2}, \epsilon\right)-\frac{1}{2}\left[\tilde{\rho}_{\phi}^{(1)}\left(N, Q^{2} / \mu^{2}, \epsilon\right)\right]^{2}\right)\right. \\
\left.-\gamma\left(\left(Q^{2} / \mu^{2}\right)^{\epsilon}, \epsilon\right) \frac{\partial}{\partial \ln Q^{2}} \tilde{\rho}_{\phi}^{(1)}\left(N, Q^{2} / \mu^{2}, \epsilon\right)\right\} .
\end{array}
$$

Of course, unlike for Drell-Yan [11], the second order corrections to Higgs production have not yet been calculated. The above results can easily be inverted to derive $W_{\phi}^{(1,2)}(z, 1, \epsilon)$, if the functions $\rho_{\phi}^{(1,2)}$ are known. The full functions $W_{\phi}^{(1,2)}\left(z, \xi^{2} / \mu^{2}, \epsilon\right)$ may then be constructed by reexpanding the running coupling $\alpha\left(\xi^{2} / \mu^{2}, \alpha\left(\mu^{2}\right), \epsilon\right)$ in $\alpha\left(\mu^{2}\right)$, using eq. (27) with $\lambda=\xi^{2} / \mu^{2}$,

$$
\begin{aligned}
& W_{\phi}^{(1)}\left(z, \xi^{2} / \mu^{2}, \epsilon\right)=\left(\frac{\mu^{2}}{\xi^{2}}\right)^{\epsilon} W_{\phi}^{(1)}(z, 1, \epsilon), \\
& W_{\phi}^{(2)}\left(z, \xi^{2} / \mu^{2}, \epsilon\right)=\left(\frac{\mu^{2}}{\xi^{2}}\right)^{2 \epsilon} W_{\phi}^{(2)}(z, 1, \epsilon)+\gamma\left(\left(\xi^{2} / \mu^{2}\right)^{\epsilon}, \epsilon\right)\left(\frac{\mu^{2}}{\xi^{2}}\right)^{\epsilon} W_{\phi}^{(1)}(z, 1, \epsilon) .
\end{aligned}
$$

In this way the one-loop coefficients $W_{\phi}^{(1)}$ can be straightforwardly determined from eqs. (18,19). They may be written as

$$
W_{\phi}^{(1)}(z, 1, \epsilon)=\delta(1-z) f_{\phi}^{(1)}(\epsilon)+z^{\epsilon}\left(\frac{g^{(1)}(z, \epsilon)}{(1-z)^{1+2 \epsilon}}\right)_{+}+h^{(1)}(z, \epsilon)
$$

where the coefficient functions $f_{\phi}^{(1)}, g^{(1)}, h^{(1)}$ are regular functions of their arguments at $z=1$. In the present case they are given by

$$
\begin{aligned}
f_{h, H}^{(1)}(\epsilon) & =-C_{A} \epsilon\left(\frac{11}{6 \epsilon}+\frac{203}{36}+\frac{\pi^{2}}{3}\right), \\
f_{A}^{(1)}(\epsilon) & =f_{h, H}^{(1)}(\epsilon)-2 C_{A} \epsilon, \\
g^{(1)}(z, \epsilon) & =C_{A}\left(1+z^{4}+(1-z)^{4}\right), \\
h^{(1)}(z, \epsilon) & =C_{A} \epsilon \frac{11}{6} z^{\epsilon}(1-z)^{3-2 \epsilon} .
\end{aligned}
$$


Given the limitations of the factorization theorem near the edge of phase space [22], from which the evolution equation (21) is derived [14], we might be tempted to immediately discard all terms of order $1 / N$. However, we wish to be careful with these terms, and we will examine them and their relevance later in this section.

As was shown in [14], the term $\delta(1-z) f_{\phi}^{(1)}$ and the plus-distributions in eq. (33) are separately renormalization group invariant. We are therefore free to choose different functions $\nu(z)$ for these two terms in the general resummed expression eq. (25). The natural choice for the $\delta(1-z) f_{\phi}^{(1)}$ term is $\nu=1$. Changes in $\mu$ generate terms $b_{2} \ln \left(\mu^{\prime} / \mu\right)$ at higher orders. The $\lambda$ integral may then be carried out explicitly for this term in eq. (25). For the plus-distribution term however, the natural choice is $\nu(z)=(1-z)^{2}$, just as for Drell-Yan. Then, using $\xi=(1-z) \mu$ in eq. (32), we absorb the factor $(1-z)^{-2 \epsilon}$ in (33) into the boundary of the $\lambda$ integral in eq. (25), involving only the running coupling. The term $h^{(1)}$ is of $\mathcal{O}\left(1 / N^{4}\right)$ and will be neglected.

We now turn our attention to the function $g^{(1)}(z, \epsilon)$ in (36). We have some freedom in its treatment, as different choices will only differ by constants or in $O(\ln N / N)$. However, we will argue that among all such terms the ones generated by $g^{(1)}(z, \epsilon)$ are universal and can legitimately be included in the resummed expression. To exhibit the importance of the different treatments of the residue function $g^{(1)}(z, \epsilon)$ we choose three schemes which probe the full range of possibilities. After rescaling to incorporate the factor $(1-z)^{-2 \epsilon}$ and combining the plus distribution with the Mellin transform in eq. (25), we see that the relevant function to approximate is $\left(z^{N-1}-1\right) g^{(1)}(z, \epsilon)$ [We are neglecting the coefficient $z^{\epsilon}$, because it is contributing at $\mathcal{O}(1 / N)$.]. The three schemes are defined by

$$
\begin{gathered}
\text { scheme } \alpha: \frac{1}{C_{A}}\left(z^{N-1}-1\right) g^{(1)}(z, \epsilon) \rightarrow\left(z^{N-1}-1\right) 2 \\
\text { scheme } \beta: \frac{1}{C_{A}}\left(z^{N-1}-1\right) g^{(1)}(z, \epsilon) \rightarrow\left(z^{N-1}-1\right) 2-(1-z)\left(2 z^{2}-4 z-2 z^{3}\right) \\
\text { scheme } \gamma: \frac{1}{C_{A}}\left(z^{N-1}-1\right) g^{(1)}(z, \epsilon) \rightarrow\left(z^{N-1}-1\right) 2-(1-z)\left(2 z^{2}-4 z-2 z^{3}\right) \\
-4 z^{N-1}(1-z) .
\end{gathered}
$$

The minimal scheme $\alpha$ involves replacing $g^{(1)}(z, \epsilon)$ simply by $g^{(1)}(1, \epsilon)$, scheme $\beta$ includes all terms of $\mathcal{O}(1)$ in the exponent, whereas scheme $\gamma$ includes in addition some $O\left(\ln ^{i} N / N\right)$ terms in the exponent. Using the one-loop evolution kernel $W_{\phi}^{(1)}$ we can now construct the resummed expressions for the Higgs production correction factor in the three schemes. However, these expressions are still divergent for $\epsilon \rightarrow 0$. The divergences are cancelled by mass factorization and renormalization for which we choose the $\overline{\mathrm{MS}}$ scheme [23] throughout this paper. For this purpose we need the resummed $\overline{\mathrm{MS}}$ gluon distribution [24]. To one loop accuracy it may be written as 
14.24

$$
\begin{aligned}
\tilde{\phi} \frac{(1)}{\mathrm{MS}}\left(N, \frac{M^{2}}{\mu^{2}}, \alpha\left(\mu^{2}\right), \epsilon\right) & =\exp \left[-\int_{0}^{M^{2}} \frac{d \mu^{\prime 2}}{\mu^{\prime 2}} \Gamma_{g g}^{(1)}\left(N, \alpha_{s}\left(\mu^{\prime 2}\right)\right)\right] \\
& =\bar{\phi} \frac{(1)}{\mathrm{MS}}\left(N, \frac{M^{2}}{\mu^{2}}, \alpha\left(\mu^{2}\right), \epsilon\right) z_{0}\left(\frac{M^{2}}{\mu^{2}}, \alpha\left(\mu^{2}\right), \epsilon\right)
\end{aligned}
$$

where

$$
\begin{aligned}
\bar{\phi}_{\overline{\mathrm{MS}}}^{(1)} & =\exp \left[\frac{C_{A}}{2} \int_{0}^{1} d z\left(\frac{z^{N-1}-1}{1-z}\right)\left(1+z^{4}+(1-z)^{4}\right) \int_{0}^{M^{2} / \mu^{2}} \frac{d \lambda}{\lambda} \alpha\left(\lambda, \alpha\left(\mu^{2}\right), \epsilon\right)\right] \\
& \times \exp \left[-\frac{11}{6} \frac{C_{A}}{2} \int_{0}^{M^{2} / \mu^{2}} \frac{d \lambda}{\lambda} \alpha\left(\lambda, \alpha\left(\mu^{2}\right), \epsilon\right)\right]
\end{aligned}
$$

and

$$
z_{0}\left(\frac{M^{2}}{\mu^{2}}, \alpha\left(\mu^{2}\right), \epsilon\right)=\exp \left[b_{2} \int_{0}^{M^{2} / \mu^{2}} \frac{d \lambda}{\lambda} \alpha\left(\lambda, \alpha\left(\mu^{2}\right), \epsilon\right)\right],
$$

where $M$ is the mass factorization scale. As is well-known, the one-loop anomalous dimension $\Gamma_{g g}^{(1)}\left(N, \alpha_{s}\right)$ is derived from the residue of the collinear singularity in the gluon operator matrix element. The function $z_{0}$ is related to that component of the residue which is proportional to the one loop coefficient of the QCD $\beta$-function. The strong coupling in the LO cross section (7) has been left unrenormalized so far. The renormalization of this bare coupling is now performed in the $\overline{\mathrm{MS}}$ scheme, via the replacement

$$
\begin{aligned}
\alpha_{s, B} & =\alpha_{s}\left(R^{2}\right)\left(R^{2}\right)^{\epsilon} Z_{\alpha}\left(\frac{R^{2}}{\mu^{2}}, \alpha_{s}\left(\mu^{2}\right), \epsilon\right) \\
& =\alpha_{s}\left(R^{2}\right)\left(R^{2}\right)^{\epsilon} \exp \left[\int_{0}^{R^{2} / \mu^{2}} \frac{d \lambda}{\lambda}\left\{b_{2} \alpha\left(\lambda, \alpha\left(\mu^{2}\right), \epsilon\right)+b_{3} \alpha^{2}\left(\lambda, \alpha\left(\mu^{2}\right), \epsilon\right)+\ldots\right\}\right]
\end{aligned}
$$

where we explicitly show the renormalization scale $R$. Thus the renormalization factorizes into several pieces according to the perturbative expansion of the QCD $\beta$ function,

$$
\alpha_{s, B}=\alpha_{s}\left(R^{2}\right)\left(R^{2}\right)^{\epsilon} z_{0}\left(\frac{R^{2}}{\mu^{2}}, \alpha_{s}\left(\mu^{2}\right), \epsilon\right) z_{1}\left(\frac{R^{2}}{\mu^{2}}, \alpha_{s}\left(\mu^{2}\right), \epsilon\right) \ldots
$$

where

$$
z_{1}\left(\frac{R^{2}}{\mu^{2}}, \alpha\left(\mu^{2}\right), \epsilon\right)=\exp \left[b_{3} \int_{0}^{R^{2} / \mu^{2}} \frac{d \lambda}{\lambda} \alpha\left(\lambda, \alpha\left(\mu^{2}\right), \epsilon\right)\right] .
$$

Note that the above form of the $Z$-factor for the strong coupling constant, which is completely factorized from the correction factor, is very similar in form to the $\delta(1-z)$ piece of the $\overline{\mathrm{MS}}$ density, and we may thus combine the overall coupling constant renormalization with the mass factorization procedure. Restricting ourselves 
to next-to-leading order (i.e. putting $z_{1}=1$ ) and choosing $R=M$ one finds that simultaneous mass factorization and renormalization of the bare coupling in eq. ([) leads to

$$
\rho_{\phi}\left(N, Q^{2} / M^{2}, \alpha\left(M^{2}\right)\right)=\frac{\rho_{\phi}\left(N, Q^{2} / \mu^{2}, \alpha\left(\mu^{2}\right), \epsilon\right)}{\left[\bar{\phi}\left(\frac{1)}{\mathrm{MS}}\left(N, M^{2} / \mu^{2}, \alpha\left(\mu^{2}\right), \epsilon\right)\right]^{2}\right.} .
$$

Note that the dependence on the dimensional regularization scale $\mu$ drops out on the 1.h.s. Because $\rho_{\phi}$ is infrared safe, we can return to four dimensions, and we find

$$
\begin{aligned}
\rho_{h, H}^{\alpha}\left(N, \frac{Q^{2}}{M^{2}}, \alpha\left(M^{2}\right)\right)= & \exp \left[-C_{A} \int_{0}^{1} d z \frac{z^{N-1}-1}{1-z} 2 \int_{(1-z)^{2}}^{1} \frac{Q^{2}}{M^{2}} \frac{d \lambda}{\lambda} \alpha\left(\lambda, \alpha\left(M^{2}\right), \epsilon\right)\right] \\
\times & \exp \left\{\alpha\left(Q^{2}\right) C_{A}\left[\pi^{2} / 3+203 / 36-11 / 6 \ln \left(\frac{Q^{2}}{M^{2}}\right)\right]\right. \\
& \left.\quad-11 / 12 \alpha^{2}\left(Q^{2}\right) C_{A} b_{2} \ln ^{2}\left(\frac{Q^{2}}{M^{2}}\right)\right\}, \\
\rho_{h, H}^{\beta}\left(N, \frac{Q^{2}}{M^{2}}, \alpha\left(M^{2}\right)\right)= & \rho_{h, H}^{\alpha}\left(N, \frac{Q^{2}}{M^{2}}, \alpha\left(M^{2}\right)\right) \\
\times & \exp \left[-2 C_{A} \int_{0}^{1} d z\left(2 z-z^{2}+z^{3}\right) \int_{(1-z)^{2}}^{1} \frac{Q^{2}}{M^{2}} \frac{d \lambda}{\lambda} \alpha\left(\lambda, \alpha\left(M^{2}\right), \epsilon\right)\right], \\
\rho_{h, H}^{\gamma}\left(N, \frac{Q^{2}}{M^{2}}, \alpha\left(M^{2}\right)\right)= & \rho_{h, H}^{\beta}\left(N, \frac{Q^{2}}{M^{2}}, \alpha\left(M^{2}\right)\right) \\
\times & \exp \left[+4 C_{A} \int_{0}^{1} d z z^{N-1} \int_{(1-z)^{2} \frac{Q^{2}}{M^{2}}}^{1} \frac{d \lambda}{\lambda} \alpha\left(\lambda, \alpha\left(M^{2}\right), \epsilon\right)\right], \\
\rho_{A}^{\alpha, \beta, \gamma}\left(N, \frac{Q^{2}}{M^{2}}, \alpha\left(M^{2}\right)\right)= & \rho_{h, H}^{\alpha, \beta, \gamma}\left(N, \frac{Q^{2}}{M^{2}}, \alpha\left(M^{2}\right)\right) \times \exp \left[2 C_{A} \alpha\left(Q^{2}\right)\right] .
\end{aligned}
$$

A few remarks are in order. First, the above expressions are formally not well defined because the integration paths in the exponent traverse a singularity, related to an infrared renormalon. Our purpose in this paper is however not the numerical evaluation of the resummed cross section; we consider the resummed formulae to be a generating functional for an approximation to the QCD perturbation series, rather than its approximate sum.

The second remark is that one may try to incorporate (a part of) the two loop evolution kernel. Some of the NNLO terms in the exact calculation can be inferred using renormalization group methods, such as used for Drell-Yan in Ref. [25]. A straightforward calculation along these lines leads to

$$
W_{\phi}^{(2)}(z, 1, \epsilon)=z(1-z)^{-4 \epsilon} P_{g g}^{(1)}(z)+\ldots
$$

where $P_{g g}^{(1)}$ is the two-loop component of the Altarelli-Parisi gluon-to-gluon splitting function [26]. Moreover, the mass factorization and renormalization of the overall 
coupling in the Born cross section can be carried out in a similar fashion as at one loop, leading to the modified $\overline{\mathrm{MS}}$ distribution (for $R=M$ )

$\bar{\phi}_{\overline{\mathrm{MS}}}^{(2)}\left(N, \frac{M^{2}}{\mu^{2}}, \alpha\left(\mu^{2}\right), \epsilon\right)=\phi \frac{(2)}{\mathrm{MS}}\left(N, \frac{M^{2}}{\mu^{2}}, \alpha\left(\mu^{2}\right), \epsilon\right) z_{0}^{-1}\left(\frac{M^{2}}{\mu^{2}}, \alpha\left(\mu^{2}\right), \epsilon\right) z_{1}^{-1}\left(\frac{M^{2}}{\mu^{2}}, \alpha\left(\mu^{2}\right), \epsilon\right) \ldots$

where $\phi \frac{(2)}{\mathrm{MS}}$ denotes the resummed $\overline{\mathrm{MS}}$ distribution based on the two-loop anomalous dimension $\Gamma_{g g}^{(2)}$ 26]. However, we stress that in this paper we restrict ourselves to examining the consequences of resummation with the one-loop kernel $W_{\phi}^{(1)}$. 回

There are several arguments supporting the inclusion of $\ln ^{i} N / N$ terms in the resummation via the $\gamma$-scheme. In momentum space such terms correspond either to the usual plus-distributions, which are already included, or to terms $\ln ^{i}(1-z)$, see the Appendix. The latter are usually discarded in Sudakov resummation procedures. Their inclusion extends the resummation formalism to incorporate subleading, in the sense that they are down by a power of $N$ compared to the plus distributions, divergent contributions for $z \rightarrow 1$ in addition to the plus distributions.

Our first observation is that those $\ln ^{i}(1-z)$ terms which are included by using scheme $\gamma$ originate from the Altarelli-Parisi splitting function, as we have demonstrated above. In distinction to the plus-distributions, which have the same origin, they are not enhanced by the infrared eikonal factor $1 /(1-z)$, hence they are essentially of a collinear nature. Their origin thus implies that they are universal and independent of the hard process. A second observation is that the integral in the exponent in eq. (48), which generates these logarithms, is partly composed of the full $\overline{\mathrm{MS}}$ distribution (40), which is an exact result, without any soft gluon approximation. A third supporting argument can be obtained from existing NNLO $(\overline{\mathrm{MS}}$ scheme) calculations for the $q \bar{q}$ channel in the Drell-Yan reaction [11] and the nonsinglet structure function $F_{2}$ [27 in deep-inelastic scattering. We are able to derive the leading $\ln ^{i}(1-z)$ terms and those subleading terms which are related to the running coupling at NNLO using the resummed formulae for the hard parts for these reactions, given in [12,14] (e.g. for Drell-Yan see eq. (5.18) and the analogous DIS result obtained from renormalizing eq. (5.13) in [14]).

Numerical consequences of including the $\ln ^{i}(1-z)$ terms will be presented in the next section for both Drell-Yan and Higgs boson production at the LHC.

\section{Two- and Three Loop Results}

In this section we examine the quality and the scheme dependence of our resummed formulae. We do this by expanding our results for the correction factors of Higgs boson

\footnotetext{
${ }^{6}$ Except for a $b_{2} \ln \left(\mu^{\prime} / \mu\right)$ term at NNLO.
} 
production and the analogous expression for the $q \bar{q}$ channel in Drell-Yan (eq. (5.18) in [14]), in various schemes $(\alpha, \beta, \gamma)$ to NLO and NNLO in order to compare with the exact results of Refs. [5] and [11]. All comparisons will be made for the LHC. We will find that for both reactions scheme $\gamma$ reproduces the exact results remarkably well, much better than the more conventional schemes $\alpha$ and $\beta$ at low Higgs $/ \gamma^{*}$ masses. We further derive the scale-dependent logarithms in the NNLO expansion from our resummed expressions. Here also the agreement is reasonable.

From the NNLO expansion of our resummed formulae for Higgs production, we can obtain the first indication on the importance of the NNLO contributions. As mentioned earlier, these estimates are important because the NLO K-factor is sizable. A full numerical study of the resummed cross sections, which requires a regularization of the renormalon singularity [28], will be published elsewhere [29].

Analogous to eq. (15) the renormalized correction factor $\rho_{\phi}$ of eqs. (46-49) may be expanded as

$$
\rho_{\phi}\left(z, Q^{2} / M^{2}, \alpha\right)=\sum_{n=0}^{\infty} \alpha^{n}\left(M^{2}\right) \rho_{\phi}^{(n)}\left(z, Q^{2} / M^{2}\right)
$$

We begin with the NLO expansion. Using the Mellin transform formulae of the Appendix and the methods of [30], we find that the $O(\alpha)$ terms, upon inversion to momentum space [keeping the renormalization/factorization scale $M^{2}$ different from $\left.Q^{2}\right]$ are given by

$$
\begin{array}{ll}
\rho_{h, H}^{\alpha(1)}\left(z, \frac{Q^{2}}{M^{2}}\right) & =C_{A}\left\{4 \mathcal{D}_{1}(z)+2 \mathcal{D}_{0}(z) L_{M}+\left(\frac{\pi^{2}}{3}+\frac{203}{36}-\frac{11}{6} L_{M}\right) \delta(1-z)\right\}, \\
\rho_{h, H}^{\beta(1)}\left(z, \frac{Q^{2}}{M^{2}}\right) & =C_{A}\left\{4 \mathcal{D}_{1}(z)+2 \mathcal{D}_{0}(z) L_{M}+\frac{\pi^{2}}{3} \delta(1-z)\right\}, \\
\rho_{h, H}^{\gamma(1)}\left(z, \frac{Q^{2}}{M^{2}}\right)=\rho_{h, H}^{\beta(1)}\left(z, \frac{Q^{2}}{M^{2}}\right)-C_{A}\left\{8 \mathcal{E}_{1}(z)\right\}, & \quad \text { [for all three schemes], } \\
\rho_{A}^{(1)}\left(z, \frac{Q^{2}}{M^{2}}\right)=\rho_{h, H}^{(1)}\left(z, \frac{Q^{2}}{M^{2}}\right)+2 C_{A} \delta(1-z) \quad
\end{array}
$$

where we use the notation

$$
\mathcal{D}_{i}(z)=\left[\frac{\ln ^{i}(1-z)}{1-z}\right]_{+} \quad, \quad \mathcal{E}_{i}(z)=\ln ^{i}(1-z) \quad, \quad L_{M}=\ln \left(Q^{2} / M^{2}\right) .
$$

With the results above we construct the correction factors according to eq. (52) for the three schemes $\alpha, \beta$ and $\gamma$ so that we can compare with exact results. Similarly one may obtain results to $O\left(\alpha^{2}\right)$. We find

$$
\begin{aligned}
\rho_{h, H}^{\alpha(2)}\left(z, Q^{2} / M^{2}\right) & =C_{A}\left\{8 C_{A} \mathcal{D}_{3}(z)+\left(-4 b_{2}+12 C_{A} L_{M}\right) \mathcal{D}_{2}(z)\right. \\
& +\left(\frac{203}{9} C_{A}-8 C_{A} \zeta_{2}-4 b_{2} L_{M}-\frac{22}{3} C_{A} L_{M}+4 C_{A} L_{M}^{2}\right) \mathcal{D}_{1}(z)
\end{aligned}
$$




$$
\begin{aligned}
& +\left(16 C_{A} \zeta_{3}-4 C_{A} \zeta_{2} L_{M}+\frac{203}{18} C_{A} L_{M}-b_{2} L_{M}^{2}-\frac{11}{3} C_{A} L_{M}^{2}\right) \mathcal{D}_{0}(z) \\
& +\left(\frac{203}{18} C_{A} \zeta_{2}+6 C_{A} \zeta_{2}^{2}-12 C_{A} \zeta_{4}+\frac{41209}{2592} C_{A}\right. \\
& +8 C_{A} \zeta_{3} L_{M}-2 \zeta_{2} b_{2} L_{M}-\frac{203}{36} b_{2} L_{M}-\frac{11}{3} C_{A} \zeta_{2} L_{M}-\frac{2233}{216} C_{A} L_{M} \\
& \left.\left.+\frac{121}{72} C_{A} L_{M}^{2}-2 C_{A} \zeta_{2} L_{M}^{2}+\frac{11}{12} b_{2} L_{M}^{2}\right) \delta(1-z)\right\}, \\
& \rho_{h, H}^{\beta(2)}\left(z, Q^{2} / M^{2}\right)=C_{A}\left\{8 C_{A} \mathcal{D}_{3}(z)+\left(-4 b_{2}+12 C_{A} L_{M}\right) \mathcal{D}_{2}(z)\right. \\
& +\left(-8 C_{A} \zeta_{2}-4 b_{2} L_{M}+4 C_{A} L_{M}^{2}\right) \mathcal{D}_{1}(z) \\
& +\left(16 C_{A} \zeta_{3}-4 C_{A} \zeta_{2} L_{M}-b_{2} L_{M}^{2}\right) \mathcal{D}_{0}(z) \\
& +\left(6 C_{A} \zeta_{2}^{2}-12 C_{A} \zeta_{4}-\frac{2909}{216} b_{2}-2 \zeta_{2} b_{2} L_{M}\right. \\
& \left.\left.+8 C_{A} \zeta_{3} L_{M}-2 C_{A} \zeta_{2} L_{M}^{2}\right) \delta(1-z)\right\}, \\
& \rho_{h, H}^{\gamma(2)}\left(z, Q^{2} / M^{2}\right)=\rho_{h, H}^{\beta(2)}\left(z, Q^{2} / M^{2}\right)+C_{A}\left\{-16 C_{A} \mathcal{E}_{3}(z)\right. \\
& +\left(8 b_{2}+8 C_{A}-24 C_{A} L_{M}\right) \mathcal{E}_{2}(z) \\
& \left.+\left(16 C_{A} \zeta_{2}+8 b_{2} L_{M}+8 C_{A} L_{M}-8 C_{A} L_{M}^{2}\right) \mathcal{E}_{1}(z)\right\}, \\
& \rho_{A}^{\alpha(2)}\left(z, Q^{2} / M^{2}\right)=\rho_{h, H}^{\alpha(2)}\left(z, Q^{2} / M^{2}\right)+C_{A}\left\{8 C_{A} \mathcal{D}_{1}(z)+4 C_{A} \mathcal{D}_{0}(z) L_{M}\right. \\
& \left.+\left(4 C_{A} \zeta_{2}-\frac{11}{3} C_{A} L_{M}+\frac{239}{18} C_{A}-2 b_{2} L_{M}\right) \delta(1-z)\right\}, \\
& \rho_{A}^{\beta(2)}\left(z, Q^{2} / M^{2}\right)=\rho_{h, H}^{\beta(2)}\left(z, Q^{2} / M^{2}\right)+C_{A}\left\{8 C_{A} \mathcal{D}_{1}(z)+4 C_{A} \mathcal{D}_{0}(z) L_{M}\right. \\
& \left.+\left(4 C_{A} \zeta_{2}+2 C_{A}-2 b_{2} L_{M}\right) \delta(1-z)\right\}, \\
& \rho_{A}^{\gamma(2)}\left(z, Q^{2} / M^{2}\right)=\rho_{h, H}^{\gamma(2)}\left(z, Q^{2} / M^{2}\right)+C_{A}\left\{8 C_{A} \mathcal{D}_{1}(z)+4 C_{A} \mathcal{D}_{0}(z) L_{M}\right. \\
& \text { - } \left.16 C_{A} \mathcal{E}_{1}(z)+\left(4 C_{A} \zeta_{2}+2 C_{A}-2 b_{2} L_{M}\right) \delta(1-z)\right\} \text {. }
\end{aligned}
$$

In constructing the scale logarithms in the above expressions we have used the replacement

$$
\alpha\left(Q^{2}\right)=\alpha\left(M^{2}\right)-\alpha\left(M^{2}\right)^{2} b_{2} L_{M}
$$

in the last exponents of eqs. (46,49). We note that this effectively amounts to including one term from the two-loop Sudakov evolution kernel $W_{\phi}^{(2)}$. This term can be derived from the one-loop evolution kernel using the renormalization group, see the discussion below eq. (37). In Fig. 团 we present the correction factors for SM Higgs production at the LHC, which coincide with the correction factors of MSSM scalar Higgs boson production for small $\operatorname{tg} \beta$, and in Fig. 5 for MSSM pseudoscalar Higgs production, where we have identified $M^{2}=Q^{2}$. For all results in this section we used the CTEQ4M parton densities [13], a two-loop running coupling constant, with $\Lambda \frac{(5)}{\mathrm{MS}}=202 \mathrm{MeV}$ for the NLO and NNLO quantities and CTEQ4L densities with a LO strong coupling constant $\left(\Lambda_{\mathrm{LO}}^{(5)}=181 \mathrm{MeV}\right)$ for the LO quantities.

In Figs. Ha and 5a the "partonic" K-factors, obtained from folding the correction 
factors $\rho_{\phi}$ with NLO parton densities and using a NLO strong coupling for all orders of the cross sections, are presented. For comparison we show in Figs. $4 \mathrm{~b}$ and $5 \mathrm{~b}$ the corresponding NLO "hadronic" K-factors [which include the small contributions from $\kappa_{\phi}$ ] normalized to the LO cross sections evolved with LO parton densities and $\alpha_{s}$. Whereas the former indicate the effect of the higher order corrections to the partonic cross section, the latter exhibit the convergence of the perturbative approach to the physical (hadronic) quantities. We observe from Figs. 4 and 5 that at NLO scheme $\gamma$ reproduces the exact NLO calculation almost exactly for the full range of the SM Higgs mass $M_{H} \gtrsim 65 \mathrm{GeV}$ and the MSSM Higgs masses $M_{\phi} \gtrsim 45 \mathrm{GeV}$, whereas the schemes $\alpha$ and $\beta$ agree with the exact result only for $M_{\phi} \gg 1 \mathrm{TeV}$ (the agreement of scheme $\alpha$ in the intermediate Higgs mass range is accidental). Moreover, note that the NNLO corrections to the partonic cross sections in scheme $\gamma$ are still significant. Full NNLO predictions for hadronic cross sections require NNLO parton densities, which are not yet available. At NLO, a significant reduction of the hadronic K-factors compared to the partonic K-factors can be read off from Figs. $4 \mathrm{~b}$ and $5 \mathrm{~b}$, indicating a more reliable perturbative QCD expansion contrary to what Figs. Ha and 5a suggest. We point out that should the size of the NNLO corrections to the physical cross section warrant concern about the convergence of the perturbative approach, our resummation method can provide a tool to control such large corrections.

Besides the three-loop anomalous dimension, the determination of NNLO densities requires NNLO calculations for the physical quantities included in a global fit. However, there are presently only a few exact NNLO calculations available [11,27.31. An approximate way to proceed might be provided by the use of NNLO expansions of resummed cross sections, which at NLO have to approximate the exact results reliably. For future high-energy hadron colliders we expect this to require the inclusion of the novel subleading contributions that have been discussed in our analysis. Once approximate NNLO results have been obtained for several processes, e.g. heavy flavor production at the LHC, and the three-loop anomalous dimensions for the NNLO evolution of the parton densities have been calculated, a global fit of approximate NNLO parton densities can be performed. The same procedure could of course also be followed at the resummed level.

To investigate the reliability of scheme $\gamma$ at NNLO we confront in Fig. 6 the approximate partonic K-factor, using NLO parton densities and strong coupling in all expressions, for the Drell-Yan $q \bar{q}$ production channel at the LHC as a function of the off-shell photon mass $Q$ with the exact calculation of Ref. [11]. We do this at NLO and NNLO in schemes $\beta$ and $\gamma$. The expressions used are given in eqs. (A.77) and (A.78) of the Appendix. We observe that for scheme $\gamma$ the agreement is again excellent at NLO. As for the Higgs case, this is perhaps not so surprising, since the NLO answer is to a large extent included in the evolution kernel. However, note that the agreement is remarkably good even at NNLO. Clearly the $\ln ^{i}(1-z)$ terms are 

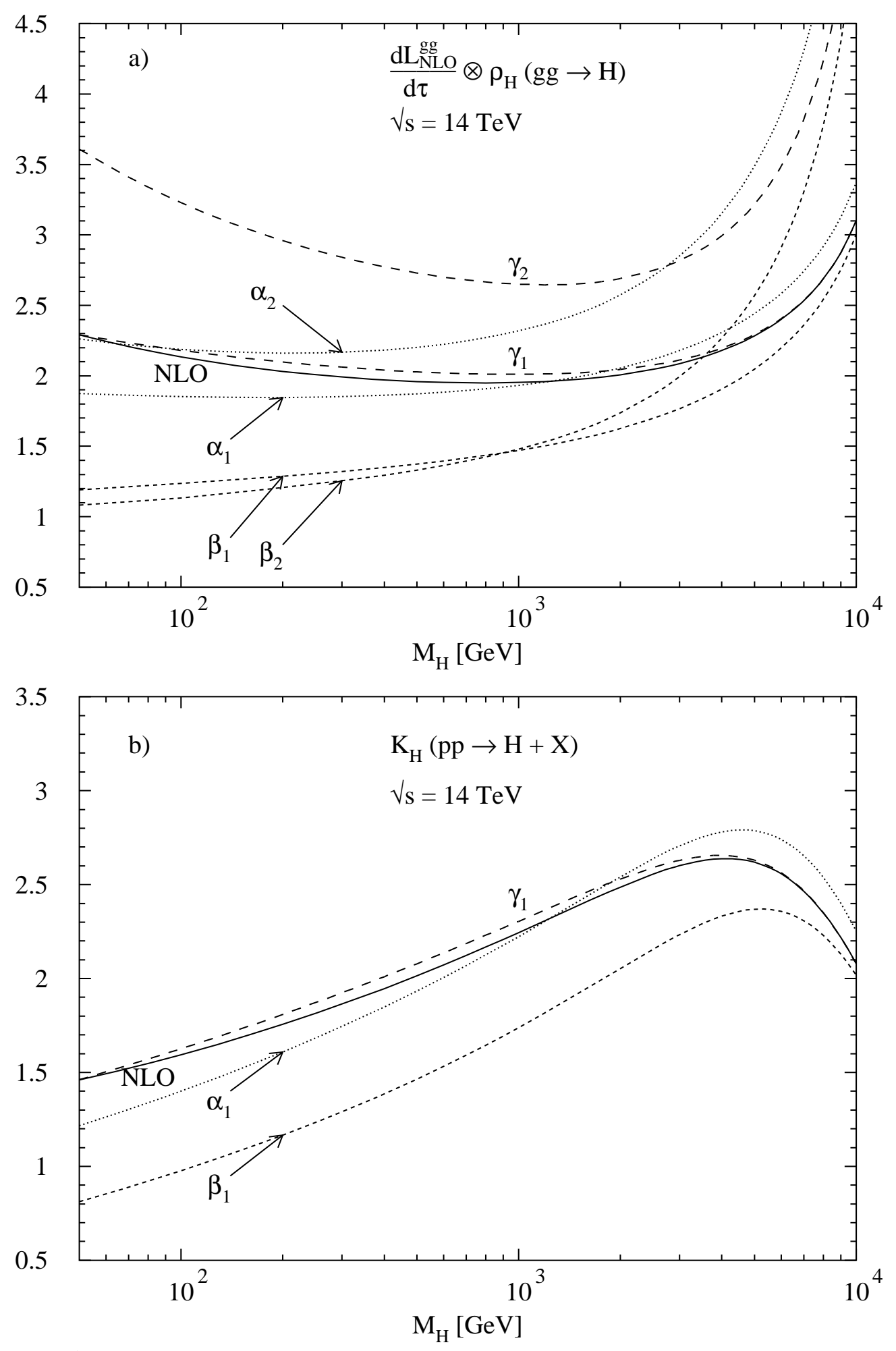

Figure 4: a) Exact and approximate two- and three-loop partonic K-factors, convoluted with the NLO gluon-gluon luminosity $d \mathcal{L}_{N L O}^{g g} / d \tau$, in the heavy top-mass limit. The results for the three different schemes are presented as a function of the scalar Higgs mass $M_{H}$, using NLO CTEQ4M parton densities [15] and $\alpha_{s}\left[\Lambda \frac{(5)}{\mathrm{MS}}=202 \mathrm{MeV}\right]$. b) Hadronic NLO K-factor using LO CTEQ4L parton densities [13] and $\alpha_{s}\left[\Lambda_{\mathrm{LO}}^{(5)}=181\right.$ $\mathrm{MeV}]$ for the LO cross section and including the NLO contributions from $\kappa_{H}$. 

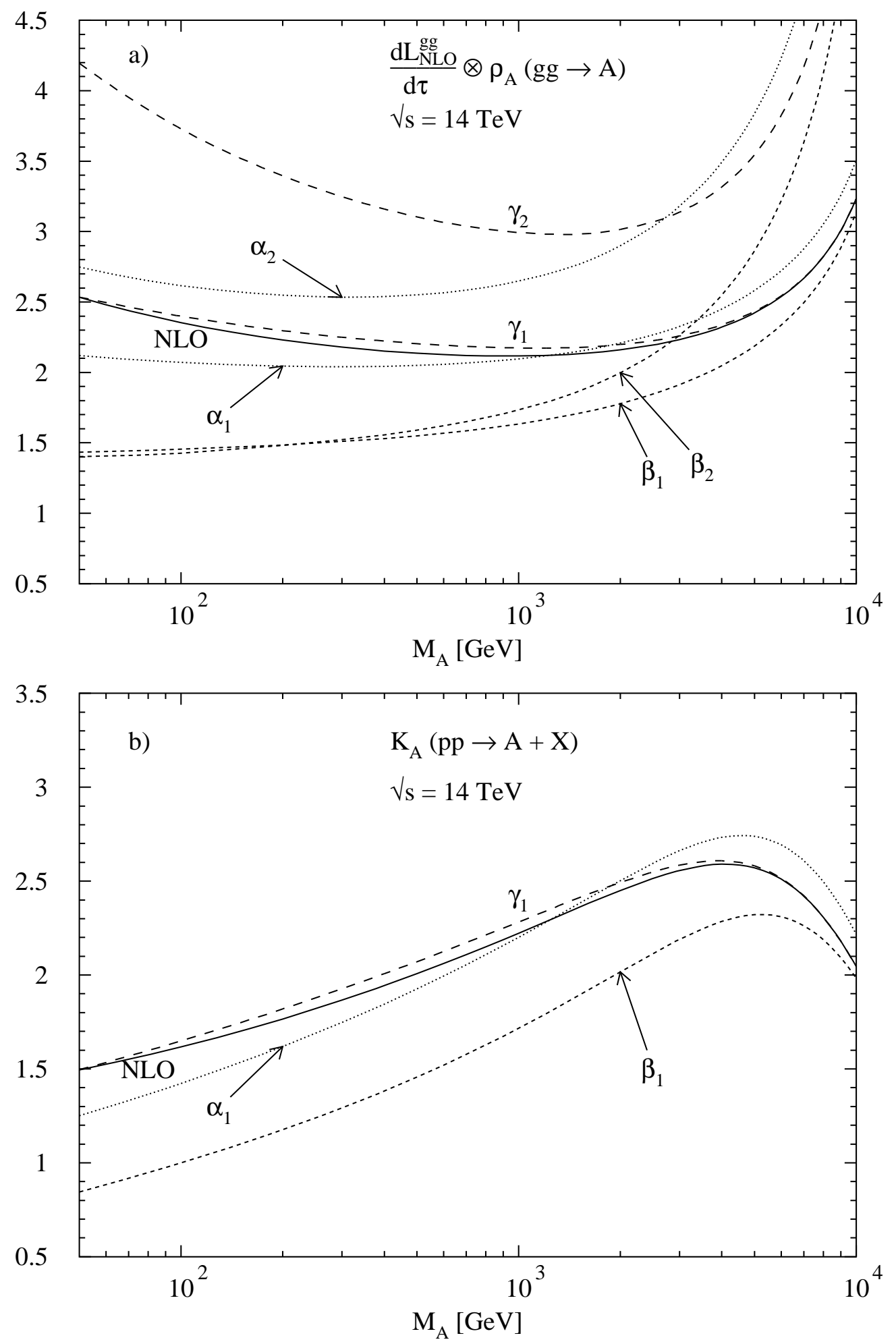

Figure 5: As in the previous figure, but now for the pseudoscalar Higgs in the MSSM for small $\operatorname{tg} \beta$. 
dominant in this order as well. In scheme $\alpha$ we found at NLO a similar accidental agreement with the exact result, as in the Higgs case. In Fig. 6 we show the NNLO result for scheme $\alpha$, which fails to approximate the exact NNLO curve, in contrast with scheme $\gamma$. Note that scheme $\beta$ fails at medium and low $Q^{2}$. The impressive agreement in NNLO, in combination with the general arguments given at the end of the previous section, gives us confidence to assert that the NNLO expansion for Higgs production in scheme $\gamma$ will be close to the exact value.

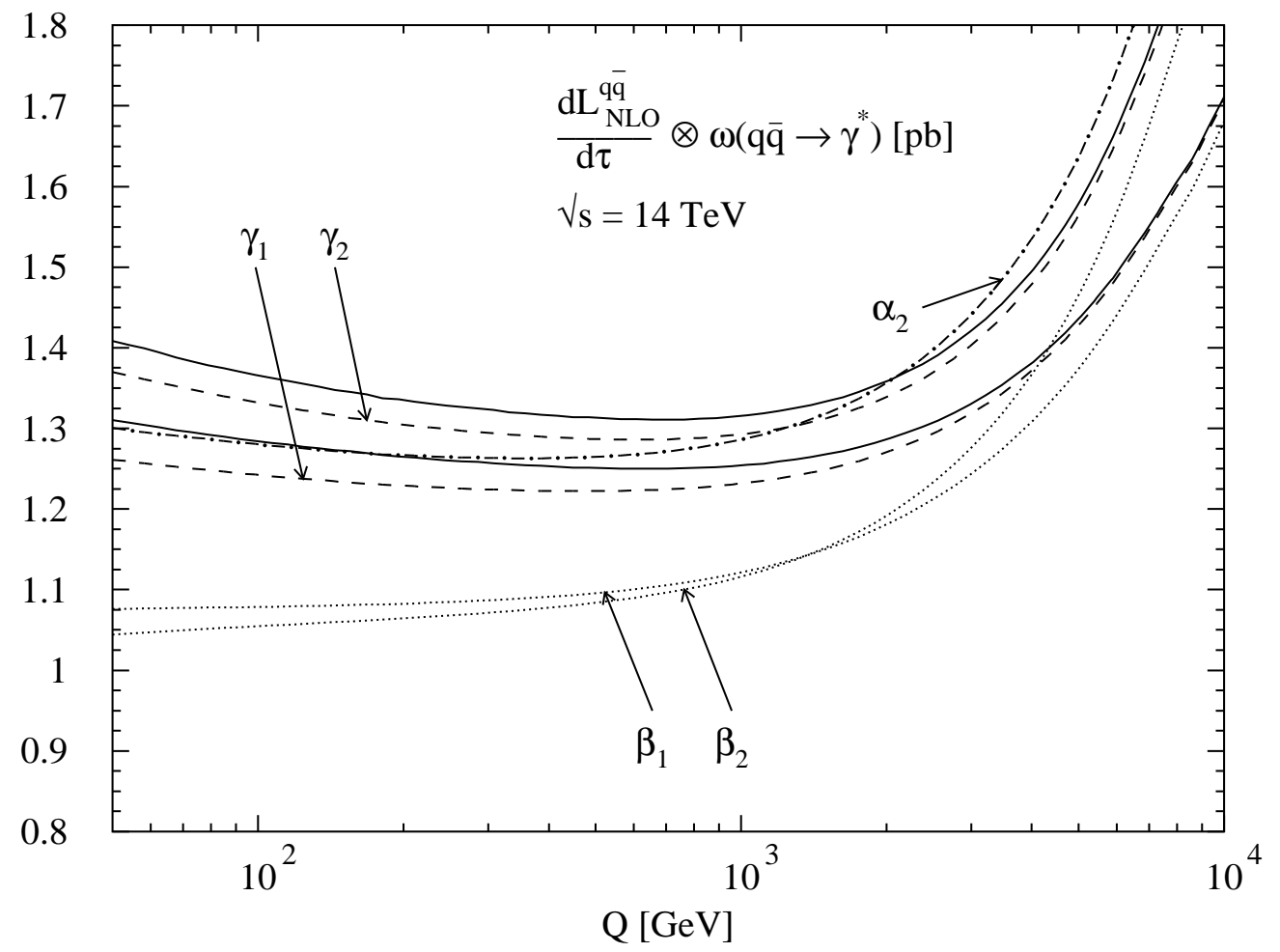

Figure 6: Exact and approximate one- and two-loop partonic K-factors, using NLO CTEQ4M parton densities [13] and strong coupling $\left[\Lambda \frac{(5)}{\mathrm{MS}}=202 \mathrm{MeV}\right]$ in all orders of the cross section, for Drell-Yan, in three different schemes, as a function of the $\gamma^{*}$ mass $Q$. The lower solid line is the exact $N L O$ result, in the $q \bar{q}$ channel, and the upper solid line is the NNLO one.

Next we investigate the consequences of the scale logarithms for Higgs production. It was found in Ref.[5] that the scale dependence of the NLO cross section is still a monotonous function of the scales. In view of the outstanding agreement of the exact results with our approximate ones in scheme $\gamma$ for $M^{2}=Q^{2}$ for the $\tau=Q^{2} / S$ dependence, we may use the same results to examine the scale dependence at NNLO. In fact, from arguments such as given in [25], one may deduce that eqs. (58-63) approximate the exact scale dependent terms very well, the only term lacking being proportional to the two-loop anomalous dimension eq. (50), which we have omitted. Again we use the Drell-Yan case to gauge the quality of scheme $\gamma$ in describing the 
scale dependence. This is done in Fig. 7 for two values of the $\gamma^{*}$ mass $Q$. To obtain the best approximation, we have defined the curve labelled $\gamma_{2}$, the NNLO approximation in scheme $\gamma$, by adding the NNLO term of the expanded resummed exponential to the exact NLO result, rather than the $\gamma_{1}$ curve. We see that at NLO and NNLO scheme $\gamma$ again describes the scale dependence quite reasonably. Again, for both the exact and approximate expressions a consistent analysis requires the use of NNLO parton densities in determining the scale dependence, but these are not available yet. (It was shown in 11] that for the full NNLO Drell-Yan cross section, including other production channels, there is a strong indication, albeit based on NLO parton densities, that the scale dependence is significantly reduced compared to NLO).

In Fig. 8 we present the scale dependence for SM Higgs production. The curve labelled $\gamma_{1}$ includes the sum of the approximate term $\rho_{H}^{\gamma(1)}$ of eq. (55) and the NLO contribution to $\kappa_{H}$ of eq. (13) for the $g g$ initial state. The approximate NNLO result, labelled $\gamma_{2}$, has been obtained by adding the NNLO term $\rho_{H}^{\gamma(2)}$ of eq. (60) and the corresponding contributions up to NNLO to $\kappa_{H}$ of eq. (13) to the exact NLO result [this significantly improves the approximation similar to the Drell-Yan process]. The curves labelled NNLO include the full NNLO scale dependence in the partonic cross section, which has been obtained from the exact NLO result by means of renormalization group methods, neglecting quarks at all stages. This curve has been normalized to the $\gamma_{2}$ curve at $\xi=1$. We observe that the NLO term of scheme $\gamma$ deviates from the exact NLO slightly at large scales and significantly for small scales. This is caused by terms of $\mathcal{O}(1 / N)$, which have been neglected. At NNLO there is a strong indication for a significant stabilization of the theoretical prediction for the total Higgs production cross section at the LHC. There are deviations between the NNLO and $\gamma_{2}$ curves at small and large scales, which are again due to terms of $\mathcal{O}(1 / N)$ that have been neglected in scheme $\gamma$. We found similar results to hold for the MSSM pseudoscalar Higgs case.

Finally, let us comment on the phenomenological implications of our results for the Higgs K-factor at NNLO. When the NLO corrections to the Higgs production cross section were calculated both for the infinite mass limit [6] and for the general massive case [5] it was found that the ratio of the NLO cross section to the LO one could be larger than two if one used NLO parton densities and strong coupling for both cross sections at LO and NLO. In order to estimate the increase in size of the QCD corrected physical cross section the hadronic K-factor has to be defined by including the corresponding cross sections evaluated with parton densities and strong coupling at the same order, i.e. LO cross sections with LO quantities and NLO with NLO quantities. This hadronic NLO K-factor amounts to 1.5-2.0 in the phenomenologically relevant Higgs mass range. This indicates that the procedure to predict with increasing accuracy the physical cross section, by including higher order corrections consistently in all quantities entering the factorization theorem, seems to 

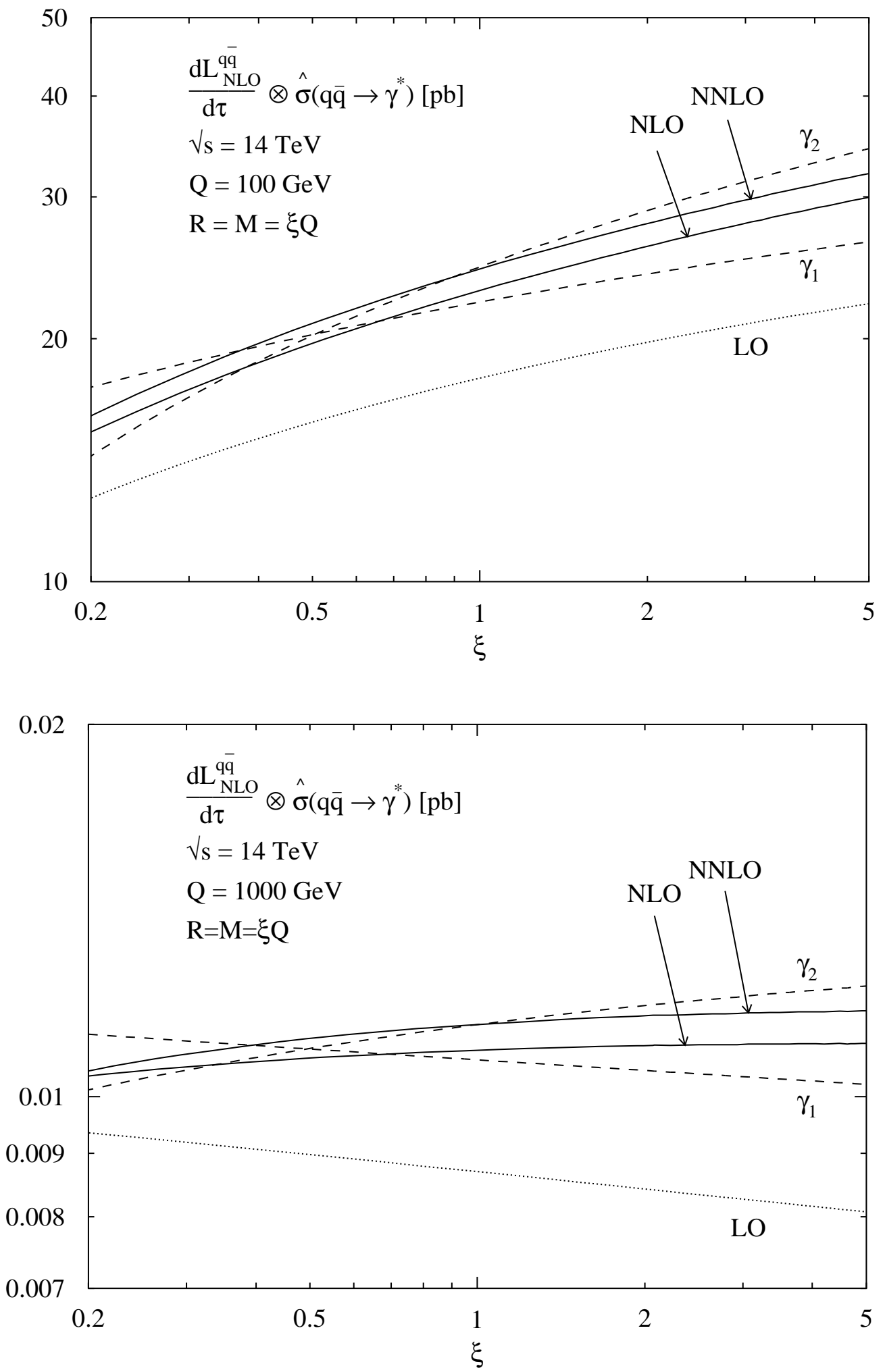

Figure 7: Scale dependence of the Drell-Yan cross section for two values of the $\gamma^{*}$ mass $Q$. The solid lines represent the exact calculation at $N L O$ and $N N L O$ and the dotted line the LO one. NLO CTEQ4M parton densities [13] and strong coupling $\left[\Lambda \frac{(5)}{\mathrm{MS}}=202 \mathrm{MeV}\right]$ have been used. 

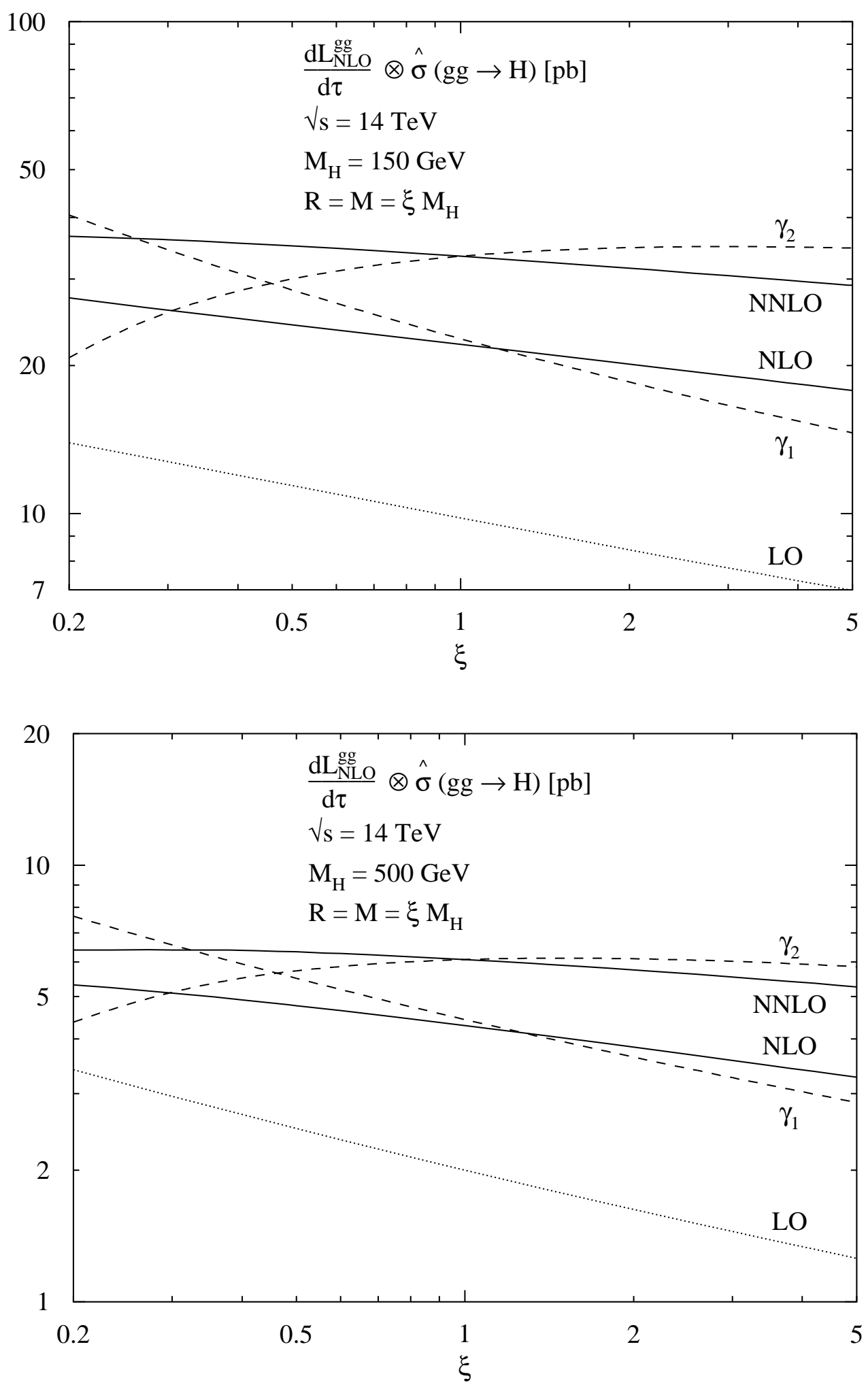

Figure 8: Scale dependence of the Higgs production cross section for two values of the Higgs mass $M_{H}$. NLO CTEQ4M parton densities 105$]$ and strong coupling $\left[\Lambda \frac{(5)}{\mathrm{MS}}=202\right.$ $\mathrm{MeV}]$ have been used in all expressions, so that the $N N L O$ results do not correspond to the physical NNLO cross sections. 
converge better than one might conclude from Figs. Fa and 5a. A consistent NNLO hadronic K-factor requires NNLO strong coupling and parton densities, which are not yet available, but one might expect a further reduction from them. However, as long as we do not know the parton densities beyond NLO, we observe from Figs. Ha and 5a that NNLO corrections to the partonic cross sections are sizable. Our demonstration that the bulk of the corrections originates from soft and collinear gluon radiation, and the fact that they can be resummed analytically, provides then a different way to organize the perturbative expansion in the phenomenologically relevant Higgs mass range: one may redefine the original QCD perturbation series by rewriting it as the product of our resummed expression, times a new series. Due to the excellent approximation of the original series by the expanded resummed series, the new series is expected to be very well behaved perturbatively. This is of course the standard method for making sense of perturbative QCD near the edges of phase space, where QCD corrections are large. However, the result can now be extended much further away from threshold due to the inclusion of the novel subleading contributions. Note that this procedure would also require resummed parton densities. We furthermore note that the large size of the corrections compared to Drell-Yan is partly due to the fact that for every power of $\alpha_{s}$ a color factor $C_{A}=3$ appears. We have seen that for Drell-Yan in the $\overline{\mathrm{MS}}$ scheme, where the corresponding color factor is $C_{F}=4 / 3$ but the analytical structure of the soft gluon corrections is quite similar, the corrections are considerably smaller. The same phenomenon was observed for the case of resummed heavy quark production in Ref. [32]. These color factors are correctly included in the resummed formulae.

As remarked earlier, the evaluation of the resummed series has its own subtleties, related to the appearance of the infrared renormalon. For its treatment there have recently been a number of proposals [28], which we will not discuss here. We anticipate that the resummed Higgs production cross section will be quite sensitive to the details of handling the renormalon, due the large color factor in the exponent [32.

\section{Conclusions}

In this paper we have performed the all order resummation of soft gluon effects in Higgs production both for the Standard Model and its minimal supersymmetric extension, to next-to-leading logarithmic accuracy. We have extended the usual resummation formalism to include logarithms which, although integrable, diverge in the partonic cross section near the edge of phase space. By expanding our resummed results to NLO and NNLO, and using the Drell-Yan process for comparison, we have shown that this extension expands the applicability of resummation efforts into the phenomenologically relevant Higgs boson production range at the LHC. An accurate 
assessment of the expected Higgs production rate is of paramount importance for the LHC physics programme. However, a physical prediction of the NNLO cross section requires knowledge of NNLO parton densities, which is not yet available. Clearly, in this regard, it would be interesting to investigate the applicability of our extended formalism to many other QCD production processes with potentially large K-factors at NLO, e.g. heavy quark production [33] both at the Tevatron and the LHC, or to revisit the Drell-Yan process for phenomenological studies along the lines of [34].

\section{Acknowledgements}

We would like to thank K. Chetyrkin, S.A. Larin, P. Nason, J. Smith, G. Sterman and P.M. Zerwas for helpful conversations. We also thank W. van Neerven and P. Rijken for providing the fortran code of the NNLO Drell-Yan process. E.L. would like to thank the Columbia University theory group and M.K. the CERN Theory Division for hospitality while this work was being completed.

\section{Appendix A: Useful formulae}

In this appendix we collect some useful formulae used in section 2 . We begin by extending the Mellin transform table of Ref. [35] up to $O(1 / N)$. Define

$$
I_{n}(N)=\int_{0}^{1} d x x^{N-1}\left[\frac{\ln ^{n}(1-x)}{1-x}\right]_{+} .
$$

For the lowest four values of $n$ this integral is, up to $O(1 / N)$

$$
\begin{aligned}
I_{0}(N) & =-\ln \tilde{N}+\frac{1}{2} \frac{1}{N} \\
I_{1}(N) & =\frac{1}{2} \ln ^{2} \tilde{N}+\frac{1}{2} \zeta_{2}-\left(\frac{1}{2} \ln \tilde{N}+\frac{1}{2}\right) \frac{1}{N} \\
I_{2}(N) & =-\frac{1}{3} \ln ^{3} \tilde{N}-\zeta_{2} \ln \tilde{N}-\frac{2}{3} \zeta_{3}+\left(\frac{1}{2} \ln ^{2} \tilde{N}+\frac{1}{2} \zeta_{2}+\ln \tilde{N}\right) \frac{1}{N} \\
I_{3}(N) & =\frac{1}{4} \ln ^{4} \tilde{N}+\frac{3}{2} \zeta_{2} \ln ^{2} \tilde{N}+2 \zeta_{3} \ln \tilde{N}+\frac{3}{4} \zeta_{2}^{2}+\frac{3}{2} \zeta_{4} \\
& +\left(-\frac{1}{2} \ln ^{3} \tilde{N}-\frac{3}{2} \zeta_{2} \ln \tilde{N}-\zeta_{3}-\frac{3}{2} \ln ^{2} \tilde{N}-\frac{3}{2} \zeta_{2}\right) \frac{1}{N}
\end{aligned}
$$

with $\tilde{N}=N e^{\gamma_{E}}$ and $\gamma_{E}$ denoting the Euler constant. Define also

$$
J_{n}(N)=\int_{0}^{1} d x x^{N-1} \ln ^{n}(1-x)
$$

For the lowest four values of $n$ this integral is, up to $O(1 / N)$

$$
J_{0}(N)=\frac{1}{N}
$$




$$
\begin{aligned}
& J_{1}(N)=-\frac{\ln \tilde{N}}{N} \\
& J_{2}(N)=\frac{\ln ^{2} \tilde{N}}{N}+\frac{\zeta_{2}}{N} \\
& J_{3}(N)=-\frac{\ln ^{3} \tilde{N}}{N}-3 \zeta_{2} \frac{\ln \tilde{N}}{N}-2 \frac{\zeta_{3}}{N}
\end{aligned}
$$

Next we present the NNLO perturbative expansions of the resummed hard part $\omega_{q \bar{q}}$ of the $\overline{\mathrm{MS}}$ Drell-Yan cross section in two schemes, defined in analogy to eq. (38). The relevant function to approximate here is $\left(z^{N-1}-1\right) g_{\mathrm{DY}}^{(1)}(z, \epsilon)$ with $g_{\mathrm{DY}}^{(1)}(z, \epsilon)=$ $C_{F}\left(1+z^{2}\right)$. The schemes $\alpha, \beta$ and $\gamma$ are defined by the replacements

$$
\begin{aligned}
\text { scheme } \alpha: \frac{1}{C_{F}}\left(z^{N-1}-1\right) g_{\mathrm{DY}}^{(1)}(z, \epsilon) & \rightarrow\left(z^{N-1}-1\right) 2 \\
\text { scheme } \beta: \frac{1}{C_{F}}\left(z^{N-1}-1\right) g_{\mathrm{DY}}^{(1)}(z, \epsilon) & \rightarrow\left(z^{N-1}-1\right) 2+(1-z)(1+z) \\
\text { scheme } \gamma: \frac{1}{C_{F}}\left(z^{N-1}-1\right) g_{\mathrm{DY}}^{(1)}(z, \epsilon) & \rightarrow\left(z^{N-1}-1\right) 2+(1-z)(1+z) \\
& -2 z^{N-1}(1-z) .
\end{aligned}
$$

We find the results

$$
\begin{aligned}
\omega_{\alpha, q \bar{q}}^{\overline{\mathrm{MS}}}\left(z, Q^{2} / M^{2}\right) & =\delta(1-z)+\alpha\left(M^{2}\right) C_{F}\left\{4 \mathcal{D}_{1}(z)+2 \mathcal{D}_{0}(z) L_{M}\right. \\
+ & \left.\left(2 \zeta_{2}-\frac{1}{2}\right) \delta(1-z)\right\}+\alpha\left(M^{2}\right)^{2} C_{F}\left\{8 C_{F} \mathcal{D}_{3}(z)+\left(-4 b_{2}\right.\right. \\
+ & \left.12 C_{F} L_{M}\right) \mathcal{D}_{2}(z)+\left(-8 C_{F} \zeta_{2}-2 C_{F}-4 b_{2} L_{M}+4 C_{F} L_{M}^{2}\right) \mathcal{D}_{1}(z) \\
+ & \left(16 C_{F} \zeta_{3}-4 C_{F} \zeta_{2} L_{M}-b_{2} L_{M}^{2}-C_{F} L_{M}\right) \mathcal{D}_{0}(z) \\
+ & \left(6 C_{F} \zeta_{2}^{2}-12 C_{F} \zeta_{4}-C_{F} \zeta_{2}+C_{F} \frac{1}{8}+\frac{1}{2} b_{2} L_{M}-2 \zeta_{2} b_{2} L_{M}\right. \\
+ & \left.\left.8 C_{F} \zeta_{3} L_{M}-2 C_{F} \zeta_{2} L_{M}^{2}\right) \delta(1-z)\right\} \\
\omega_{\beta, q \bar{q}}^{\mathrm{MS}}\left(z, Q^{2} / M^{2}\right) & =\delta(1-z)+\alpha\left(M^{2}\right) C_{F}\left\{4 \mathcal{D}_{1}(z)+2 \mathcal{D}_{0}(z) L_{M}\right. \\
+ & \left.\left(2 \zeta_{2}-4+\frac{3}{2} L_{M}\right) \delta(1-z)\right\} \\
+ & \alpha\left(M^{2}\right)^{2} C_{F}\left\{8 C_{F} \mathcal{D}_{3}(z)+\left(-4 b_{2}+12 C_{F} L_{M}\right) \mathcal{D}_{2}(z)\right. \\
+ & \left(-8 C_{F} \zeta_{2}-16 C_{F}+6 C_{F} L_{M}-4 b_{2} L_{M}+4 C_{F} L_{M}^{2}\right) \mathcal{D}_{1}(z) \\
+ & \left(16 C_{F} \zeta_{3}-4 C_{F} \zeta_{2} L_{M}-8 C_{F} L_{M}-b_{2} L_{M}^{2}+3 C_{F} L_{M}^{2}\right) \mathcal{D}_{0}(z) \\
+ & \left(6 C_{F} \zeta_{2}^{2}-12 C_{F} \zeta_{4}-\frac{15}{2} b_{2}-8 C_{F} \zeta_{2}+8 C_{F}-6 C_{F} L_{M}\right. \\
+ & 4 b_{2} L_{M}-2 \zeta_{2} b_{2} L_{M}+3 C_{F} \zeta_{2} L_{M}+8 C_{F} \zeta_{3} L_{M} \\
& \left.\left.-\frac{3}{4} b_{2} L_{M}^{2}-2 C_{F} \zeta_{2} L_{M}^{2}+\frac{9}{8} C_{F} L_{M}^{2}\right) \delta(1-z)\right\} \\
\omega_{\gamma, q \bar{q}}^{\mathrm{MS}}\left(z, Q^{2} / M^{2}\right) & =\omega_{\beta, q \bar{q}}\left(z, Q^{2} / M^{2}\right)+
\end{aligned}
$$




$$
\begin{aligned}
& +\alpha\left(M^{2}\right) C_{F}\left\{-4 \mathcal{E}_{1}(z)\right\} \\
& +\alpha\left(M^{2}\right)^{2} C_{F}\left\{-8 C_{F} \mathcal{E}_{3}(z)+\left(4 b_{2}+8 C_{F}-12 C_{F} L_{M}\right) \mathcal{E}_{2}(z)\right. \\
& \left.+\left(16 C_{F}+8 C_{F} \zeta_{2}+2 C_{F} L_{M}+4 b_{2} L_{M}-4 C_{F} L_{M}^{2}\right) \mathcal{E}_{1}(z)\right\} .
\end{aligned}
$$

\section{References}

[1] P.W. Higgs, Phys. Rev. Lett. 12 (1964) 132 and Phys. Rev. 145 (1966) 1156; F. Englert and R. Brout, Phys. Rev. Lett. 13 (1964) 321; G.S. Guralnik, C.R. Hagen and T.W. Kibble, Phys. Rev. Lett. 13 (1964) 585.

[2] J.-F. Grivaz, Proceedings, International Europhysics Conference on High Energy Physics, Brussels 1995.

[3] N. Cabibbo, L. Maiani, G. Parisi and R. Petronzio, Nucl. Phys. B158 (1979) 295; M. Chanowitz, M. Furman and I. Hinchliffe, Phys. Lett. B78 (1978) 285; R.A. Flores and M. Sher, Phys. Rev. D27 (1983) 1679; M. Lindner, Z. Phys. C31 (1986) 295; M. Sher, Phys. Rep. 179 (1989) 273; A. Hasenfratz, K. Jansen, C. Lang, T. Neuhaus and H. Yoneyama, Phys. Lett. B199 (1987) 531; J. Kuti, L. Liu and Y. Shen, Phys. Rev. Lett. 61 (1988) 678; M. Lüscher and P. Weisz, Nucl. Phys. B318 (1989) 705.

[4] H. Georgi, S. Glashow, M. Machacek and D.V. Nanopoulos, Phys. Rev. Lett. 40 (1978) 692.

[5] D. Graudenz, M. Spira and P. M. Zerwas, Phys. Rev. Lett. 70 (1993) 1372; M. Spira, A. Djouadi, D. Graudenz and P. M. Zerwas, Phys. Lett. B318 (1993) 347; M. Spira, A. Djouadi, D. Graudenz and P.M. Zerwas, Nucl. Phys. B453 (1995) 17.

[6] A. Djouadi, M. Spira and P. M. Zerwas, Phys. Lett. B264 (1991) 440; S. Dawson, Nucl. Phys. B359 (1991) 283; R. P. Kauffman and W. Schaffer, Phys. Rev. D49 (1994) 551; S. Dawson and R.P. Kauffman, Phys. Rev. D49 (1994) 2298.

[7] Y. Okada, M. Yamaguchi and T. Yanagida, Prog. Theor. Phys. 85 (1991) 1; H. Haber and R. Hempfling, Phys. Rev. Lett. 66 (1991) 1815; J. Ellis, G. Ridolfi and F. Zwirner, Phys. Lett. B257 (1991) 83; R. Barbieri, F. Caravaglios and M. Frigeni, Phys. Lett. B258 (1991) 167; M. Carena, J. Espinosa, M. Quiros and C.E.M. Wagner, Phys. Lett. B355 (1995) 209; M. Carena, M. Quiros and C.E.M. Wagner, Nucl. Phys. B461 (1996) 407. 
[8] Z. Kunszt and F. Zwirner, Nucl. Phys. B385 (1992) 3; V. Barger, M. Berger, S. Stange and R. Phillips, Phys. Rev. D45 (1992) 4128; H. Baer, M. Bisset, C. Kao and X. Tata, Phys. Rev. D46 (1992) 1067; J. F. Gunion and L. Orr, Phys. Rev. D46 (1992) 2052; J. F. Gunion, H. E. Haber and C. Kao, Phys. Rev. D46 (1992) 2907.

[9] S. Dawson, A. Djouadi and M. Spira, Phys. Rev. Lett. 77 (1996) 16.

[10] I. Hinchliffe and S. Novaes, Phys. Rev. D38 (1988) 3475; R.P. Kauffman, Phys. Rev. D44 (1991) 1415; Phys. Rev. D45 (1992) 1512; C.-P. Yuan, Phys. Lett. B283 (1992) 395; C. Kao, Phys. Lett. B328 (1994) 420.

[11] R. Hamberg, T. Matsuura and W.L. van Neerven, Nucl. Phys. B359 (1991) 343;

E. Zijlstra and W.L. van Neerven, Nucl. Phys. B382 (1992) 11.

[12] G. Sterman, Nucl. Phys. B281 (1987) 310; S. Catani and L. Trentadue, Nucl. Phys. B327 (1989) 323; Nucl. Phys. B353 (1991) 183; H. Contopanagos and G. Sterman, Nucl. Phys. B400 (1993) 211; Nucl. Phys. B419 (1994) 77; L. Alvero and H. Contopanagos, Nucl. Phys. B436 (1995) 184; Nucl. Phys. B456 (1995) 497.

[13] H.L. Lai et al.(CTEQ Collab.), Phys. Rev. D55 (1997) 1280.

[14] H. Contopanagos, E. Laenen and G. Sterman, preprint CERN-TH/96-75, hepph/9604313, to appear in Nucl. Phys. B.

[15] J. Ellis, M.K. Gaillard and D.V. Nanopoulos, Nucl. Phys. B106 (1976) 292; A.I. Vainshtain, M.B. Voloshin, V.I. Sakharov and M.A. Shifman, Sov. J. Nucl. Phys. 30 (1979) 711; B.A. Kniehl and M. Spira, Z. Phys. C69 (1995) 77.

[16] T. Inami, T. Kubota and Y. Okada, Z. Phys. C18 (1983) 69.

[17] K.G. Chetyrkin, B.A. Kniehl and M. Steinhauser, preprint MPI/PhT/97-006, hep-ph/9705240.

[18] S.A. Larin, T. van Ritbergen and J.A.M. Vermaseren, Nucl. Phys. B438 (1995) 278.

[19] W. Bernreuther and W. Wetzel, Nucl. Phys. B197 (1982) 228; W. Bernreuther, Ann. Phys. 151 (1983) 127.

[20] O. Tarasov, A. Vladimirov and A. Zharkov, Phys. Lett. B93 (1980) 429.

[21] O. Tarasov, Preprint JINR P2-82-900 (1982), unpublished. 
[22] G. Sterman, in Ref. [12].

[23] W.A. Bardeen, A.J. Buras, D.W. Duke and T. Muta, Phys. Rev. D18 (1978) 3998.

[24] J.C. Collins and D.E. Soper, Nucl. Phys. B194 (1982) 445.

[25] T. Matsuura and W.L. van Neerven, Z. Phys. C38 (1988) 632; T. Matsuura, S. van der Marck and W.L. van Neerven, Phys. Lett. B211 (1988) 171; Nucl. Phys. B319 (1989) 570.

[26] E.G. Floratos, D.A. Ross and C.T. Sachrajda, Nucl. Phys. B129 (1977) 66, (E) ibid. B139 (1978) 545; Nucl. Phys. B152 (1979) 493. A. Gonzalez-Arroyo, C. Lopez and F.J. Yndurain Nucl. Phys. B153 (1979) 161; A. GonzalezArroyo and C. Lopez, Nucl. Phys. B166 (1980) 429; E.G. Floratos, P. Lacaze and C. Kounnas, Phys. Lett. B98 (1981) 225; G. Gurci, W. Furmanski and R. Petronzio, Nucl. Phys. B175 (1980) 27. W. Furmanski and R. Petronzio, Phys. Lett. B97 (1980) 437.

[27] E. Zijlstra and W.L. van Neerven, Phys. Lett. B272 (1991) 127; Nucl. Phys. B383 (1992) 525.

[28] D. Appell, P. Mackenzie and G. Sterman, Nucl. Phys. B309 (1988) 259; H. Contopanagos and G. Sterman, Ref. [12]. Y. Dokshitser, G. Marchesini and B. Webber, preprint CERN-TH/95-281,hep-ph/9512336; S. Catani, M. Mangano, P. Nason and L. Trentadue, Nucl. Phys. B478 (1996) 273.

[29] M. Krämer, E. Laenen and M. Spira, in preparation.

[30] L. Magnea, Nucl. Phys. B349 (1991) 703.

[31] E.B. Zijlstra and W.L. van Neerven, Phys. Lett. B297 (1992) 377; Nucl. Phys. B474 (1994) 61,(E) ibid. B426 (1994) 245.

[32] E. Laenen, J. Smith and W.L. van Neerven, Nucl. Phys. B369 (1992) 543.

[33] E. Laenen, J. Smith and W.L. van Neerven, in Ref. [32]; Phys. Lett. B321 (1994) 254; E.L. Berger and H. Contopanagos, Phys. Lett. B361 (1995) 115; Phys. Rev. D54 (1996) 3085; N. Kidonakis and J. Smith, Phys. Rev. D51 (1995) 6092; S. Catani, M.L. Mangano, P. Nason and L. Trentadue, in Ref. [28]; N. Kidonakis and G. Sterman, preprint ITP-SB-96-7, hep-ph/9604234; S. Mrenna and C.P. Yuan, Phys. Rev. D55 (1997) 120; N. Kidonakis, J. Smith and R. Vogt, preprint ITP-SB-96-32, hep-ph/9608343.

[34] P.J. Rijken and W.L. van Neerven, Phys. Rev. D51 (1995) 44; L. Alvero, H. Contopanagos, in Ref. [12]. 
[35] S. Catani and L. Trentadue, in Ref. 112 